\title{
Understanding unconventional routes to impurities from drugs in hydrolytic conditions
}

\author{
Jasmeen Kaur ${ }^{1}$, Yogita Bansal ${ }^{2}$, Gulshan Bansal ${ }^{3 *}$ \\ Department of Pharmaceutical Sciences and Drug Research, Punjabi University, Patiala, INDIA.
}

\begin{abstract}
Introduction: Hydrolytic degradation is the most common cause of formation of impurities or degradation products in drugs during different stages of drug product development and/or shelf life of the drug/product. Degradation products formed by hydrolysis of ester, amide, urethane, sulfonamide, sulfonate and ether linkages, and of nitrile, hydroxyl and amino groups in drugs can be conveniently predicted and identified. Many drugs are known to degrade to such expected conventional hydrolytic degradation products, and the mechanisms of such degradations are also well known and reported. However, many drugs are reported to degrade under hydrolytic conditions to products, which cannot be justified by the conventional hydrolytic reactions. Objectives: Though structures of such unconventional hydrolytic products can be characterized through different spectral techniques, but there is a need to understand the mechanisms of such unconventional hydrolytic reactions in order to help in establishing intrinsic stability characteristics of a drug. Methodology: In the present review, we have studied and critically analysed all possible reports on hydrolytic degradation of various dugs to provide a thorough insight into unconventional routes of hydrolytic degradations of drugs. The various unconventional hydrolytic reactions found responsible for degradation of drugs are classified as oxidation, dehydrogenation, coupling/condensation, N-alkylation, $\mathrm{C}-\mathrm{C}$ bond cleavage, $\mathrm{C}-\mathrm{N}$ bond cleavage, dehalogenation, cyclization, decarboxylation and hydroxylation. Discussion: Varied types of reactions under hydrolytic conditions are triggered/controlled by the nature of substituent(s) across or around the susceptible bonds/groups. The mechanisms for such unconventional hydrolytic reactions have been discussed or proposed with support from the standard literature. The contents are expected to enable an analyst and a drug formulator to predict various possible as well as seemingly improbable hydrolytic degradation products of a drug well ahead of systematic forced degradation studies.
\end{abstract}

Keywords: Forced degradation, Impurities, Hydrolysis, Conventional, Unconventional, Mechanisms.

\section{INTRODUCTION}

With the enforcement of different guidelines issued by various international drugs regulatory authorities such as WHO, USFDA, EMEA and $\mathrm{ICH}$, the control of impurities in drugs has become an integral component of drug development process. ${ }^{1-4}$ Many guidelines issued by individual countries such as Therapeutic Product Directorate (Canada) and Therapeutic Goods Administration (Australia) also recommend that impurities should be controlled in the drug products. ${ }^{5-6}$ All these guidelines require, as a part of the registration dossier of the drug product, submission of complete data of any impurity that may be inherently present or formed by degradation at any stage of the drug and/or product development. The data includes information about identification, separation and structural characterization of the impurity.

ICH guidelines have classified the impurities as organic, inorganic and residual sol-
Submission Date : 08-04-2016 Revision Date : 12-05-2016 Accepted Date :13-05-2016

DOI: 10.5530/ijper.50.3.28 Correspondence: Gulshan Bansal,

Department of Pharmaceutical Sciences and Drug Research, Punjabi University, Patiala, INDIA.

Tel. +91-175-3056255

Fax. +91-175-2283073

E-mail: gulshanbansal@ rediffmail.com; gulshan@ pbi.ac.in

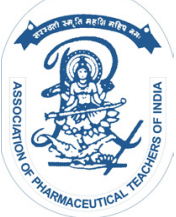

www.ijper.org 
vents. ${ }^{3,4,7,8}$ These are also classified as process related impurities (PRIs) that are incorporated in a drug substance during the synthesis and purification processes, and degradation related impurities (DRIs) that originate during formulation development, transport and/or shelf life of the product. DRIs are also termed as degradation products or degradation impurities. These arise invariably due to chemical susceptibility of different functional groups (such as amide, ester, ether, nitrile, active methylenes, nitro and other groups) present in a drug substance to varied chemical environments (such as moisture, heat, light, $\mathrm{pH}$ modulating agents, taste modifiers, antioxidants, preservatives and air) to which the drug may be exposed at different stages of product development, storage and transport. During the normal course of formulation development, and shelf life, DRIs are formed usually in trace levels. Hence, their structural characterization becomes a challenging task. ICH guideline Q1A(R2) has recommended conduct of forced degradation studies or stress studies on drug substances under different chemical environments to facilitate identification of DRIs. ${ }^{9}$ The purported aim of these studies is to generate all possible impurities that may form during shelf life of drug substance or product in a short span, and outline the degradation behaviour of the drug. These data will help in establishing intrinsic stability characteristics of the drug, developing and validating stability-indicating assay methods, and assisting a drug formulator in selection of excipients that discourage the formation of DRIs.

Hydrolysis is one of the most common causes of degradation of drug substances. Almost every drug molecule contains one or more functional group or linkage, which is susceptible to hydrolysis. It is more common in drug substances formulated as liquid orals and injectables. Nonetheless, the probability of hydrolytic degradation is equal in solid dosage forms owing to presence of some essential moisture content. Moreover, many excipients such as taste modifiers, preservatives and antioxidants can modulate $\mathrm{pH}$ of the product, which can affect the rate and extent of hydrolytic degradation. Numerous research reports on degradation of different drugs under hydrolytic conditions are available in literature. Mabey and Mill ${ }^{10}$ have reviewed hydrolytic behaviour of different classes of organic compounds under different chemical environments. Reviews of hydrolytic susceptibility of sulfonylureas, cellulose and polymers are also available in literature. ${ }^{11-13}$

In the present review, we have analysed the available literature reports on degradation behaviour of drugs in order to understand their fate under hydrolytic conditions, irrespective of their chemical and therapeutic class. These reports are classified on the basis of types of chemical reactions that are reported to be responsible for degradation of the drugs. Broadly, these reactions are classified into two categories, i.e. conventional reactions such as hydrolysis of ester, amide, ether, and other linkages, and unconventional reactions such as oxidation, dehydrogenation, dehalogenation, decarboxylation, deamination, hydroxylation, cyclization, coupling, $\mathrm{N}$-alkylation, $\mathrm{C}-\mathrm{C}$ bond cleavage, reduction, and others. The mechanism(s) for any hydrolytic degradation, wherever reported, were reviewed. The mechanisms that seemed improbable or irrational at the outset were studied critically by referring to the literature of organic chemistry. Some of the reported mechanisms did not receive any literature support. For some of such cases, alternate mechanisms were proposed with appropriate support from the literature. The reported routes/mechanisms of drug degradation are shown by bold arrows whereas the alternately proposed routes, wherever possible, are represented by dotted arrows in the same Figure. This review can be helpful in predicting the hydrolytic susceptibility of any drug before the conduct of forced degradation study.

\section{CONVENTIONAL HYDROLYTIC REACTIONS}

The amide (carboxamide and sulfonamide), ester (carboxylic ester, phosphoester, thioate ester and sulfonate ester), sulfonylurea and/or ether linkages in a drug are readily hydrolyzed in aqueous environment. However, water alone is not usually sufficient to affect these degradations due to the poor leaving ability of $-\mathrm{NH}_{2},-\mathrm{NHR}$, $-\mathrm{NR}_{2}$ and $-\mathrm{OR}$ groups. Addition of a trace amount of acid or alkali catalyzes this reaction significantly. The rate and extent of hydrolytic degradation depends invariably on $\mathrm{pH}$ of the medium (acidic, alkaline or neutral), exposure temperature and time, and nature of the other functional groups in the drug. Both amide and ester linkages are readily cleaved under hydrolytic conditions through bimolecular nucleophilic substitution $\left(\mathrm{S}_{\mathrm{N}} 2\right)$ mechanism. ${ }^{14}$

Carboxamide and sulfonamide linkages have contrasting hydrolytic susceptibilities. While carboxamide is readily cleaved in alkaline medium, sulfonamide remains relatively stable. On the other hand, carboxamide remian stable in acidic medium but sulfonamide is readily hydrolyzed. ${ }^{14}$ Nonetheless, not all sulfonamides are hydrolyzed in acidic medium. Sulfasalazine, a classical drug, is highly resistant to acidic hydrolysis. This exceptionally high stability of sulfasalazine is attributed to diazo group at para-position with respect to the sulfonyl group. This para orientation of the two groups causes 
electrons to delocalize and makes the sulfonyl sulfur resistant to nucleophilic attack by oxygen of water molecule (nucleophile). ${ }^{15}$ The nature of substituents across the C-N, S-N or C-O bond also plays an important role in deciding hydrolytic susceptibility of these bonds. Sulfonylurea derived drugs and pesticides are highly susceptible to hydrolysis especially in acidic medium. ${ }^{11}$ Glibenclamide, gliclazide, glimepiride and glipizide degrade to sulfonamide and an amine with the liberation of $\mathrm{CO}_{2}{ }^{16-19}$ This degradation has been suggested to be initiated equally through O-protonation or N-protonation. ${ }^{18}$ Drugs known to degrade due to amide and/ or ester linkages under different hydrolytic environments are given in Table 1, and structures of some of these drugs, with cleavage sites indicated by dotted lines, are given in Figure 1(a)-1(c).

An ether linkage is usually hydrolyzed under vigorous conditions, such as concentrated acids and high temperatures, to an alkyl halide and an alcohol, or two molecules of alkyl halides through unimolecular and bimolecular nucleophilic substitution $\left(\mathrm{S}_{\mathrm{N}} 1\right.$ and $\left.\mathrm{S}_{\mathrm{N}} 2\right)$ reactions. ${ }^{88}$ Many drugs degrade through ethereal cleavage even in mild acidic medium at different temperatures. Ambrisentan, ${ }^{89}$ azilsartan, ${ }^{32}$ bosentan, ${ }^{28}$ candesartan cilexetil, ${ }^{30}$ doxofylline,${ }^{39}$ duloxetine, ${ }^{90}$ etifoxine, ${ }^{91}$ etofenamate, ${ }^{46}$ idarubicin, ${ }^{92}$ lacosamide, ${ }^{57}$ NPC $1161 \mathrm{C}$ (8-aminoquinoline antimalarial drug), ${ }^{93}$ thiocolchicoside,${ }^{78}$ topiramate ${ }^{94}$ and toremifene ${ }^{95}$ are examples of such drugs (Table 2, Figure 2). Baertschi and Alsante have very clearly outlined the pathway of degradation of duloxetine due to cleavage of ether linkage, ${ }^{96}$ which is supported by Bansal et al to explain the similar degradation behaviour of toremifene. ${ }^{95}$

Conversion of a nitrile group $(\mathrm{C} \equiv \mathrm{N})$ to carboxylic acid through addition-substitution mechanism is another conventional hydrolytic reaction, which is responsible for chemical conversion of anastrazole, ${ }^{97}$ citalopram, ${ }^{98}$ saxagliptin ${ }^{99}$ and zaleplon ${ }^{100}$ (Table 2, Figure 2). Polarization of $\mathrm{C} \equiv \mathrm{N}$ bond attracts nucleophilic attack of $\mathrm{H}_{2} \mathrm{O}$ in alkaline medium to form an addition product, which tautomerizes to amide. The latter subsequently hydrolyzes to carboxylic acid depending on the type of substituent present. In case of alogliptin ${ }^{101}$ and vilazodone, ${ }^{82}$ reaction halts at the amide product. Lomustine ${ }^{102}$ degrades to secondary amines with the loss of $\mathrm{NO}_{2}$ under hydrolytic conditions (Figure 2). Some drugs containing hydroxyl group such as desvenlafaxine ${ }^{103}$ and pridinol $^{104}$ undergoes dehydration under acidic hydrolytic condition.

\section{UNCONVENTIONAL HYDROLYTIC REACTIONS}

Many drugs degrade under hydrolytic conditions to products, whose formation cannot be explained on the basis of conventional hydrolytic reactions as discussed above. Such degradation products, termed here as unconventional hydrolytic products, are reported to form by oxidation, dehydrogenation, dehalogenation, decarboxylation, deamination, hydroxylation, cyclization, coupling, $\mathrm{N}$-alkylation, and other reactions as mentioned earlier. Mechanisms of formation of only a few of such unconventional hydrolytic drug degradation products are proposed and discussed in literature. The mechanisms of formation of other such products remain elusive or unexplored. In this section, we have critically analyzed all possible reports on unconventional hydrolytic products, classified those on the basis of type of reaction, and proposed or discussed the possible mechanisms, or dissented the reported proposed routes for formation of these products.

Oxidation: Drugs belonging to chemical categories of amines, thioethers, sulfoxides and alcohols are well known to undergo oxidation under oxidative or photolytic conditions, and their mechanisms are well established. ${ }^{105}$ Some of such drugs are also reported to degrade through oxidation under hydrolytic conditions (Table 3, Figure 3), but mechanisms of such oxidative degradations remain unknown. In fact, no report has attempted to propose any possible mechanism for such hydrolytic oxidations. N-, S- or C-oxidation usually occurs under the influence of oxygen (air or atmospheric oxidation) or other oxidizing agents such as potassium permanganate, potassium dichromate and hydrogen peroxide (chemical oxidation). Under hydrolytic conditions, when no chemical oxidizing agent is added, the only possible reason of oxidation remains the air, which is usually not flushed out from the reaction medium while carrying out forced degradation studies. Therefore, this oxygen from the air in the medium seems responsible for oxidation through different mechanisms. Degradation of dexamethasone and fesoterodine due to oxidation of hydroxymethylene group can be very conveniently explained on the basis of radical mediated mechanism. Similarly, degradation of benzopyridooxathiazepine by oxidation of methylene linker to carbonyl linker can be supported on the basis of a report wherein such products are reported to form in aqueous medium but through radicals. ${ }^{106}$ Amines are also oxidized in air but through unknown radical mediated mechanisms. Now, the other perplexing question that may arise is that from where these radicals can originate in aqueous acidic or alkaline media? Usually during forced degradation studies in aqueous hydrolytic environment, dissolved oxygen and other gases are not removed with the help of nitrogen flushing. So, it may this dissolved oxygen that may generate some radicals from water and trigger these unconventional reactions. 
Table 1: Drugs degrading through hydrolysis of amide and/or ester linkages

\begin{tabular}{|c|c|c|}
\hline Drug & Hydrolytic condition(s) & Reference \\
\hline Acetazolamide & $1 \mathrm{M} \mathrm{HCl}, 24 \mathrm{~h} ; 1 \mathrm{M} \mathrm{NaOH}, 24 \mathrm{~h}$ & [20] \\
\hline Alizapride & $1 \mathrm{M} \mathrm{HCl}, 70^{\circ} \mathrm{C}, 72 \mathrm{~h} ; 0.5 \mathrm{M} \mathrm{NaOH}, 70^{\circ} \mathrm{C}, 48 \mathrm{~h}$ & [21] \\
\hline Amlodipine & $\begin{array}{l}5 \mathrm{M} \mathrm{NaOH}, 80^{\circ} \mathrm{C}, 6 \mathrm{~h} \\
1 \mathrm{M} \mathrm{HCl}, 80^{\circ} \mathrm{C}, 30 \mathrm{~m}\end{array}$ & $\begin{array}{l}{[22]} \\
{[23]}\end{array}$ \\
\hline Amtolmetin guacyl & $1 \mathrm{~N} \mathrm{HCl}, \mathrm{RT}, 60 \mathrm{~h} ; 1 \mathrm{~N} \mathrm{NaOH}, \mathrm{RT}, 1 \mathrm{~h}$ & {$[24]$} \\
\hline Benazepril & $\begin{array}{l}1 \mathrm{~N} \mathrm{HCl}, 80^{\circ} \mathrm{C}, 4 \mathrm{~h} ; 0.1 \mathrm{~N} \mathrm{NaOH}, \mathrm{RT}, 4 \mathrm{~h} ; \\
\mathrm{H}_{2} \mathrm{O}, 80^{\circ} \mathrm{C}, 4 \mathrm{~h}\end{array}$ & [25] \\
\hline Benzopyridooxathiazepine & $0.1 \mathrm{M} \mathrm{HCl}, 70^{\circ} \mathrm{C}, 60$ days & [26] \\
\hline Bicalutamide & $0.1 \mathrm{~N} \mathrm{NaOH}, 40^{\circ} \mathrm{C}, 24 \mathrm{~h}$ & [27] \\
\hline Bosentan & $0.1 \mathrm{M} \mathrm{HCl}, 85^{\circ} \mathrm{C}, 12 \mathrm{~h} ; 0.1 \mathrm{M} \mathrm{NaOH}, 85^{\circ} \mathrm{C}, 12 \mathrm{~h}$ & [28] \\
\hline Cabozantinib & $1 \mathrm{M} \mathrm{HCl}, 90^{\circ} \mathrm{C}, 30 \mathrm{~m} ; 1 \mathrm{M} \mathrm{NaOH}, 90^{\circ} \mathrm{C}, 30 \mathrm{~m}$ & [29] \\
\hline Candesartan cilexetil & $0.1 \mathrm{M} \mathrm{NaOH}, 48 \mathrm{~h}$ & [30] \\
\hline Carisbamate & $2 \mathrm{~N} \mathrm{HCl}, 60^{\circ} \mathrm{C}, 1.5 \mathrm{~h} ; 0.2 \mathrm{~N} \mathrm{NaOH}, 1 \mathrm{~h}$ & [31] \\
\hline Chlorthalidone & $0.1 \mathrm{M} \mathrm{NaOH}, 100^{\circ} \mathrm{C}, 1 \mathrm{~h}$ & [32] \\
\hline Ciclesonide & $1 \mathrm{M} \mathrm{HCl}, \mathrm{RT}, 8 \mathrm{~h} ; 0.1 \mathrm{M} \mathrm{NaOH}, \mathrm{RT}, 8 \mathrm{~h}$ & [33] \\
\hline Clopidogrel & $0.5 \mathrm{~N} \mathrm{HCl}, 80^{\circ} \mathrm{C} 12 \mathrm{~h} ; 0.1 \mathrm{~N} \mathrm{NaOH}, \mathrm{RT}, 12 \mathrm{~h} ; \mathrm{H}_{2} \mathrm{O}: \mathrm{ACN}(1: 1), 80^{\circ} \mathrm{C}, 12 \mathrm{~h}$ & [34] \\
\hline Darifenacin & $2 \mathrm{~N} \mathrm{HCl}, 60^{\circ} \mathrm{C}, 48 \mathrm{~h} ; 1 \mathrm{~N} \mathrm{NaOH}, 60^{\circ} \mathrm{C}, 48 \mathrm{~h}$ & [35] \\
\hline Darunavir & $0.5 \mathrm{~N} \mathrm{HCl}, 75^{\circ} \mathrm{C}, 48 \mathrm{~h}$ & [36] \\
\hline DDB & $2 \mathrm{M} \mathrm{HCl}, 100^{\circ} \mathrm{C}, 14 \mathrm{~h} ; 0.2 \mathrm{M} \mathrm{NaOH}, 100^{\circ} \mathrm{C}, 1 \mathrm{~h}$ & [37] \\
\hline Docetaxel & $0.1 \mathrm{~N} \mathrm{NaOH}, \mathrm{RT}, 4 \mathrm{~h}$ & [38] \\
\hline Doxofylline & $0.1 \mathrm{~N} \mathrm{HCl}, 65^{\circ} \mathrm{C}, 72 \mathrm{~h} ; 0.1 \mathrm{~N} \mathrm{NaOH}, 65^{\circ} \mathrm{C}, 72 \mathrm{~h}$ & [39] \\
\hline Dutasteride & $1 \mathrm{M} \mathrm{HCl}, 80^{\circ} \mathrm{C}, 8 \mathrm{~h} ; 1 \mathrm{M} \mathrm{NaOH}, 80^{\circ} \mathrm{C}, 4 \mathrm{~h}$ & [40] \\
\hline Efavirenz & $0.1 \mathrm{~N} \mathrm{NaOH}, \mathrm{RT}, 4 \mathrm{~h}$ & [41] \\
\hline Enalapril & $\begin{array}{l}1 \mathrm{M} \mathrm{NaOH}, 60^{\circ} \mathrm{C}, 1 \mathrm{~h} / 1 \mathrm{M} \mathrm{NaOH}, 100^{\circ} \mathrm{C}, 10 \mathrm{~m} \\
0.1 \mathrm{~N} \mathrm{HCl}, 80^{\circ} \mathrm{C}, 18 \mathrm{~h} ; 0.1 \mathrm{~N} \mathrm{NaOH}, 80^{\circ} \mathrm{C}, 3 \mathrm{~h} ; \mathrm{H}_{2} \mathrm{O}, 80^{\circ} \mathrm{C}, 2 \text { days }\end{array}$ & $\begin{array}{l}{[42]} \\
{[43]}\end{array}$ \\
\hline Eplerenone & $1 \mathrm{M} \mathrm{HCl}, 100^{\circ} \mathrm{C}, 2 \mathrm{~h} ; 1 \mathrm{M} \mathrm{NaOH}, 100^{\circ} \mathrm{C}, 2 \mathrm{~h}$ & [44] \\
\hline Eslicarbazepine & $2 \mathrm{~N} \mathrm{HCl}, \mathrm{RT}, 15 \mathrm{~m} ; 0.001 \mathrm{~N} \mathrm{NaOH}, \mathrm{RT}, 15 \mathrm{~m} ; \mathrm{H}_{2} \mathrm{O}, 60^{\circ} \mathrm{C}, 48 \mathrm{~h}$ & [45] \\
\hline Etofenamate & $10 \% \mathrm{NaOH}, 60^{\circ} \mathrm{C}, 2 \mathrm{~h}$ & [46] \\
\hline Famciclovir & $\begin{array}{l}0.1 \mathrm{M} \mathrm{HCl}, \mathrm{RT}, 3 \mathrm{~h} ; 0.005 \mathrm{M} \mathrm{NaOH} \\
\mathrm{H}_{2} \mathrm{O}, 80^{\circ} \mathrm{C}, 8 \mathrm{~h}\end{array}$ & [47] \\
\hline Fenofibrate & $0.2 \mathrm{M} \mathrm{NaOH}, 100^{\circ} \mathrm{C}, 2 \mathrm{~h}$ & [48] \\
\hline Fesoterodine & $2 \mathrm{M} \mathrm{HCl}, \mathrm{RT}, 6 \mathrm{~h} ; 0.01 \mathrm{M} \mathrm{NaOH}, \mathrm{RT}, 15 \mathrm{~m}$ & [49] \\
\hline Florfenicol & $1 \mathrm{~N} \mathrm{HCl}, 60^{\circ} \mathrm{C}, 2 \mathrm{~h} ; 1 \mathrm{~N} \mathrm{NaOH}, 60^{\circ} \mathrm{C}, 1 \mathrm{~h}$ & {$[50]$} \\
\hline Flupirtine & $1 \mathrm{M} \mathrm{HCl}, \mathrm{RT}, 24$ h; $0.01 \mathrm{M} \mathrm{NaOH}, \mathrm{RT}, 3 \mathrm{~h}$ & [51] \\
\hline Fosinopril & Varied concentrations of $\mathrm{HCl}$ and $\mathrm{NaOH}$ & [52] \\
\hline Glimepiride & $0.1 \mathrm{~N} \mathrm{NaOH}, 85^{\circ} \mathrm{C}, 72 \mathrm{~h}$ & [18] \\
\hline Indinavir & $0.1 \mathrm{~N} \mathrm{HCl}, 80^{\circ} \mathrm{C}, 24 \mathrm{~h} ; 0.1 \mathrm{~N} \mathrm{NaOH}, 80^{\circ} \mathrm{C}, 72 \mathrm{~h}$ & [53] \\
\hline Ipratropium & $5 \mathrm{~N} \mathrm{HCl}, 80^{\circ} \mathrm{C}, 30 \mathrm{~m} ; 0.1 \mathrm{~N} \mathrm{NaOH}, 60^{\circ} \mathrm{C}, 10 \mathrm{~m}$ & [54] \\
\hline Ivabradine & $1 \mathrm{~N} \mathrm{HCl} / 1 \mathrm{H}_{2} \mathrm{SO}_{4}, 80^{\circ} \mathrm{C}, 1 \mathrm{~h} ; 3 \mathrm{~N} \mathrm{NaOH}, 80^{\circ} \mathrm{C}, 48 \mathrm{~h}$ & [55] \\
\hline Lacidipine & $0.5 \mathrm{M} \mathrm{HCl}, 100^{\circ} \mathrm{C}, 30 \mathrm{~m} ; 0.1 \mathrm{M} \mathrm{NaOH}, 80^{\circ} \mathrm{C}, 30 \mathrm{~m}$ & {$[56]$} \\
\hline Lacosamide & $2 \mathrm{~N} \mathrm{HCl}, 14 \mathrm{~h} ; 1 \mathrm{~N} \mathrm{NaOH}, 2 \mathrm{~h}$ & [57] \\
\hline Larotaxel & $0.1 \mathrm{M} \mathrm{HCl}, 4 \mathrm{~h} ; 0.1 \mathrm{~N} \mathrm{NaOH}, 2 \mathrm{~h}$ & [58] \\
\hline Lopinavir & $1 \mathrm{M} \mathrm{HCl}, 80^{\circ} \mathrm{C}, 12 \mathrm{~h} ; 1 \mathrm{M} \mathrm{NaOH}, 80^{\circ} \mathrm{C}, 12 \mathrm{~h}$ & [59] \\
\hline Loratadine & $2 \mathrm{M} \mathrm{NaOH}, 100^{\circ} \mathrm{C}, 1 \mathrm{~h}$ & [60] \\
\hline Lornoxicam & $0.1 \mathrm{~N} \mathrm{HCl}, 80^{\circ} \mathrm{C}, 3 \mathrm{~h} ; 0.1 \mathrm{~N} \mathrm{NaOH}, 80^{\circ} \mathrm{C}, 1 \mathrm{~h} ; \mathrm{H}_{2} \mathrm{O}, 80^{\circ} \mathrm{C}, 3 \mathrm{~h}$ & [61] \\
\hline Lurasidone & $0.1 \mathrm{~N} \mathrm{NaOH}, 100^{\circ} \mathrm{C}, 2 \mathrm{~h}$ & [62] \\
\hline Niacinamide & $1 \mathrm{~N} \mathrm{HCl}, 80^{\circ} \mathrm{C}, 3 \mathrm{~h} ; 1 \mathrm{~N} \mathrm{NaOH}, 80^{\circ} \mathrm{C}, 3 \mathrm{~h}$ & [63] \\
\hline
\end{tabular}

Continue... 


\begin{tabular}{|c|c|c|}
\hline Drug & Hydrolytic condition(s) & Reference \\
\hline Olmesartan & $0.1 \mathrm{M} \mathrm{HCl}, 60^{\circ} \mathrm{C}, 24 \mathrm{~h} ; 0.01 \mathrm{M} \mathrm{NaOH}, \mathrm{RT}, 4 \mathrm{~h}$ & [64] \\
\hline Oxcarbazepine & $0.5 \mathrm{~N} \mathrm{NaOH}, \mathrm{RT}, 48 \mathrm{~h}$ & [65] \\
\hline Pantethine & $0.1 \mathrm{M} \mathrm{HCl}, 45^{\circ} \mathrm{C}, 6 \mathrm{~h} ; 0.01 \mathrm{M} \mathrm{NaOH}, \mathrm{RT}, 8 \mathrm{~h}$ & [66] \\
\hline Paracetamol & $0.1 \mathrm{M} \mathrm{HCl}, \mathrm{RT}, 24 \mathrm{~h} ; 0.1 \mathrm{M} \mathrm{NaOH}, \mathrm{RT}, 24 \mathrm{~h}$ & [67] \\
\hline Pentoxyfylline & $0.1 \mathrm{~N} \mathrm{NaOH}, 80^{\circ} \mathrm{C}, 4$ days & [68] \\
\hline Pentoxyverine & $1 \mathrm{M} \mathrm{HCl}, 100^{\circ} \mathrm{C}, 1 \mathrm{~h} ; 1 \mathrm{M} \mathrm{NaOH}, \mathrm{RT}, 1 \mathrm{~h}$ & [69] \\
\hline Pipenzolate & $0.1 \mathrm{M} \mathrm{HCl}, 100^{\circ} \mathrm{C}, 6 \mathrm{~h} ; 0.01 \mathrm{M} \mathrm{NaOH}, 100^{\circ} \mathrm{C}, 20 \mathrm{~m}$ & [70] \\
\hline Quinapril & $0.1 \mathrm{~N} \mathrm{HCl}, 80^{\circ} \mathrm{C}, 24 \mathrm{~h} ; 0.1 \mathrm{~N} \mathrm{NaOH}, 80^{\circ} \mathrm{C}, 24 \mathrm{~h} ; \mathrm{H}_{2} \mathrm{O}, 80^{\circ} \mathrm{C}, 24 \mathrm{~h}$ & [71] \\
\hline Ritonavir & $0.1 \mathrm{~N} \mathrm{NaOH}, \mathrm{RT}, 72 \mathrm{~h} ; \mathrm{H}_{2} \mathrm{O}, 75^{\circ} \mathrm{C}, 30 \mathrm{~h}$ & [72] \\
\hline Rivaroxaban & $0.1 \mathrm{M} \mathrm{NaOH}, 60^{\circ} \mathrm{C}, 3 \mathrm{~h}$ & [73] \\
\hline Rivastigmine & $0.5 \mathrm{~N} \mathrm{NaOH}, 48 \mathrm{~h}$ & [74] \\
\hline Ropinirole & $1 \mathrm{~N} \mathrm{HCl}, 80^{\circ} \mathrm{C}, 1 \mathrm{~h} ; 1 \mathrm{~N} \mathrm{NaOH}, 80^{\circ} \mathrm{C}, 0.5 \mathrm{~h}$ & [75] \\
\hline Sulfadimethoxine & $0.1 \mathrm{M} \mathrm{HCl}, 80^{\circ} \mathrm{C}, 3 \mathrm{~h}$ & [76] \\
\hline Tazarotene & $0.5 \mathrm{~N} \mathrm{NaOH}, \mathrm{RT}, 48 \mathrm{~h}$ & [77] \\
\hline Thiocolchicoside & $0.5 \mathrm{~N} \mathrm{HCl}, 70^{\circ} \mathrm{C}, 4 \mathrm{~h}$ & [78] \\
\hline Torasemide & $1 \mathrm{M} \mathrm{HCl}, 70^{\circ} \mathrm{C}, 24 \mathrm{~h} ; 1 \mathrm{M} \mathrm{NaOH}, 70^{\circ} \mathrm{C}, 7$ days; $\mathrm{H}_{2} \mathrm{O}, 70^{\circ} \mathrm{C}, 24 \mathrm{~h}$ & [79] \\
\hline Valsartan & $\begin{array}{l}1 \mathrm{~N} \mathrm{HCl}, 80^{\circ} \mathrm{C}, 12 \mathrm{~h} ; \mathrm{H}_{2} \mathrm{O}, 80^{\circ} \mathrm{C}, 36 \mathrm{~h} \\
2 \mathrm{~N} \mathrm{HCl}, 60^{\circ} \mathrm{C}, 1 \mathrm{~h}\end{array}$ & $\begin{array}{l}{[80]} \\
{[81]}\end{array}$ \\
\hline Vilazodone & $0.5 \mathrm{~N} \mathrm{HCl}, 80^{\circ} \mathrm{C}, 8 \mathrm{~h} ; 0.5 \mathrm{~N} \mathrm{NaOH}, 80^{\circ} \mathrm{C}, 2 \mathrm{~h}$ & [82] \\
\hline XPRIL & $1 \mathrm{M} \mathrm{HCl}, 100^{\circ} \mathrm{C}, 15 \mathrm{~m} ; 1 \mathrm{M} \mathrm{NaOH}, 100^{\circ} \mathrm{C}, 15 \mathrm{~m}$ & [83] \\
\hline YK-1101 & $1 \mathrm{M} \mathrm{HCl}, 60^{\circ} \mathrm{C}, 4 \mathrm{~h} ; 0.01 \mathrm{M} \mathrm{NaOH}, 60^{\circ} \mathrm{C}, 4 \mathrm{~h}$ & [84] \\
\hline Zofenopril & $1 \mathrm{M} \mathrm{NaOH}, 80^{\circ} \mathrm{C}, 48 \mathrm{~h}$ & [85] \\
\hline Zolmitriptan & $0.1 \mathrm{~N} \mathrm{NaOH}, \mathrm{RT}, 48 \mathrm{~h}$ & [86] \\
\hline Zolpidem & $5 \mathrm{~N} \mathrm{NaOH}, 50^{\circ} \mathrm{C}, 6 \mathrm{~h}$ & [87] \\
\hline
\end{tabular}

Base catalyzed ether hydrolysis: Cleavage of ether linkage in acidic medium is quite common and well documented, as discussed under conventional reactions. But certain drugs are reported to degrade by cleavage of ethereal linkage in alkaline medium also. Duloxetine degrades to 1-naphthol through the cleavage of aryl alkyl ether linkage in alkaline medium..$^{90}$ This degradation reaction may be mechanized as follows: Thiophene nucleus and naphthyloxy oxygen make the $\alpha$-alkyl carbon electron deficient (Figure 4). It attracts nucleophilic attack of $\mathrm{OH}^{-}$and releases naphthyloxy ion (better leaving group), which is then converted into naphthol. Bosentan is another drug, which is reported to degrade due to the loss of 2-hydroxyethyl group through the cleavage of aryl alkyl ether linkage in acidic as well as alkaline media. ${ }^{28}$ Here, it is proposed to occur as follows: In alkaline medium, hydroxyl group is ionized to form an oxide (a nucleophile), which subsequently attacks the $\alpha$-carbon resulting in formation of oxirane and phenolic product (Figure 4). This proposition is based on the reactions for formation of oxiranes. ${ }^{115,105}$ Based on these proposed mechanisms of reactions, it can be hypothesized that an aryl alkyl ether linkage, in which $\alpha$-carbon is electron deficient, may be hydrolyzed under alkaline condition also.

Coupling/Condensation: Lomustine (Figure 5) degrade under alkaline hydrolytic condition to a product, which is characterized as dimer. ${ }^{102}$ However, no explanation or possible mechanism for such an extremely unusual reaction is mentioned. The normal expected course of reaction under hydrolytic condition is hydrolysis of lomustine to a carbamic acid intermediate (CAI), which is highly unstable, and is immediately hydrolyzed to cyclohexylamine. Formation of dicyclohexyl urea (a dimer product) may be justified through bimolecular nucleophilic substitution reaction between cyclohexylamine and $\mathrm{CAI}^{1}$ or lomustine itself ${ }^{2}$ (Figure 5), wherein nucleophilic nitrogen of cyclohexylamine is attracted towards electrophilic carbonyl carbon of CAI or lomustine.

PAC-I (Figure 1(b)) is reported to degrade to a dimer product. ${ }^{116}$ Formation of this degradation product is possible only if hydrazide N-N bond is cleaved. However, we have not found any support in literature where such a cleavage is reported to occur in hydrolytic environment. Degradation of idrocilamide to a dimer 


Table 2: Drugs degrading through hydrolysis of ether, nitrile and N-nitrosoamine
groups

\begin{tabular}{|c|c|c|c|}
\hline Drug & Hydrolytic condition (s) & Oxidation type & Reference \\
\hline Benzopyridooxathiazepine & $0.1 \mathrm{M} \mathrm{HCl}, 70^{\circ} \mathrm{C}, 60$ days & C-oxidation & [26] \\
\hline Dexamethasone & $\begin{array}{c}0.01 \mathrm{~N} \mathrm{HCl}, 105^{\circ} \mathrm{C}, 16 \mathrm{~h} ; \\
0.01 \mathrm{~N} \mathrm{NaOH}, 105^{\circ} \mathrm{C}, 16 \mathrm{~h}\end{array}$ & $\begin{array}{c}\text { Oxidation of } \\
\text { hydroxymethyl }\end{array}$ & [106] \\
\hline Emtricitabine & $1 \mathrm{~N} \mathrm{HCl}, 80^{\circ} \mathrm{C}, 8 \mathrm{~h}$ & S-oxidation & [108] \\
\hline Esomeprazole & $0.1 \mathrm{M} \mathrm{HCl}, 60^{\circ} \mathrm{C}, 1 \mathrm{~h}$ & S-oxidation & [109] \\
\hline Fesoterodine & $2 \mathrm{M}, \mathrm{HCl}, \mathrm{RT}, 36 \mathrm{~h} ; 0.01 \mathrm{M} \mathrm{NaOH}, \mathrm{RT}, 15 \mathrm{~m}$ & $\begin{array}{c}\text { Oxidation of } \\
\text { hydroxymethyl }\end{array}$ & [49] \\
\hline Palonosetron & $\begin{array}{l}5 \mathrm{~N} \mathrm{HCl}, 100^{\circ} \mathrm{C}, 48 \mathrm{~h} ; \\
2 \mathrm{~N} \mathrm{NaOH}, 90^{\circ} \mathrm{C}, 60 \mathrm{~h}\end{array}$ & N-oxidation & [110] \\
\hline Prochlorperazine & $1 \mathrm{~N} \mathrm{HCl}, 80^{\circ} \mathrm{C}, 3$ days & S-oxidation & [111] \\
\hline Quinacrine & $\begin{array}{c}1 \mathrm{M} \mathrm{HCl}, 80^{\circ} \mathrm{C}, 2.5 \mathrm{~h} ; \\
1 \mathrm{M} \mathrm{NaOH}, 80^{\circ} \mathrm{C}, 2.5 \mathrm{~h} ; \\
\mathrm{H}_{2} \mathrm{O}, 80^{\circ} \mathrm{C}, 4 \mathrm{~h}\end{array}$ & N-oxidation & [112] \\
\hline Rabeprazole & $0.2 \mathrm{mM} \mathrm{HCl}, 60^{\circ} \mathrm{C}, 12 \mathrm{~h}$ & $\begin{array}{l}\text { N-oxidation } \\
\text { S-oxidation }\end{array}$ & [113] \\
\hline Tolterodine & $0.1 \mathrm{~N} \mathrm{NaOH}$, reflux, $4 \mathrm{~h}$ & N-oxidation & [114] \\
\hline
\end{tabular}


Table 4: Drugs degrading through cleavage of C-N bond under hydrolytic conditions

\begin{tabular}{|c|c|c|c|}
\hline Drug & Hydrolytic condition(s) & Reaction type & Reference \\
\hline Abacavir & $1 \mathrm{~N} \mathrm{HCl}, 80^{\circ} \mathrm{C}, 24 \mathrm{~h}$ & $\begin{array}{l}\text { Deamination, } \\
\text { Ketonization }\end{array}$ & [126] \\
\hline Acyclovir & $1 \mathrm{~N} \mathrm{NaOH}, 50^{\circ} \mathrm{C}, 12 \mathrm{~h}$ & Deamination & [127] \\
\hline Alogliptin & $1 \mathrm{M} \mathrm{HCl}, 80^{\circ} \mathrm{C}, 1.5 \mathrm{~h}$ & Ketonization & [101] \\
\hline Amlodipine & $\begin{array}{l}1 \mathrm{M} \mathrm{HCl}, 80^{\circ} \mathrm{C}, 30 \mathrm{~m} \text {; } \\
1 \mathrm{M} \mathrm{NaOH}, 80^{\circ} \mathrm{C}, 1 \mathrm{~h}\end{array}$ & Deamination & [23] \\
\hline Aripiprazole & $0.5 \mathrm{~N} \mathrm{NaOH}, 60^{\circ} \mathrm{C}, 48 \mathrm{~h}$ & Deamination & [128] \\
\hline Dipyridamole & $0.1 \mathrm{~N} \mathrm{HCl}, \mathrm{RT}, 4 \mathrm{~h}$ & Substitution & [129] \\
\hline Eberconazole & $5 \mathrm{~N} \mathrm{HCl}, 60^{\circ} \mathrm{C}, 30$ days; $5 \mathrm{~N} \mathrm{NaOH}, 60^{\circ} \mathrm{C}, 30$ days & $\begin{array}{l}\text { Substitution } \\
\text { Ketonization }\end{array}$ & [130] \\
\hline Emtricitabine & $0.1 \mathrm{~N} \mathrm{NaOH}, 80^{\circ} \mathrm{C}, 8 \mathrm{~h}$ & Deamination & [108] \\
\hline Enrofloxacin & $\begin{array}{l}5 \mathrm{~N} \mathrm{HCl}, 70^{\circ} \mathrm{C}, 1 \mathrm{~h} ; \\
5 \mathrm{~N} \mathrm{NaOH}, 70^{\circ} \mathrm{C}, 1 \mathrm{~h}\end{array}$ & $\begin{array}{l}\text { Deamination } \\
\text { Substitution }\end{array}$ & [131] \\
\hline Entecavir & $1 \mathrm{M} \mathrm{HCl}, 80^{\circ} \mathrm{C}, 36 \mathrm{~h}$ & $\begin{array}{l}\text { Deamination } \\
\text { Ketonization }\end{array}$ & [132] \\
\hline Etifoxine & $2 \mathrm{M} \mathrm{HCl}, 60 \mathrm{~h} ; 2 \mathrm{M} \mathrm{NaOH}, 120 \mathrm{~h}$ & Deamination & [91] \\
\hline Fesoterodine & $2 \mathrm{M} \mathrm{HCl}, \mathrm{RT}, 6 \mathrm{~h}$ & Deamination & [49] \\
\hline Gemifloxacin & $0.1 \mathrm{M} \mathrm{HCl}, 65^{\circ} \mathrm{C}, 72 \mathrm{~h}$ & Substitution & [133] \\
\hline Lamivudine & $\begin{array}{l}0.1 \mathrm{~N} \mathrm{HCl}, 80^{\circ} \mathrm{C}, 48 \mathrm{~h} ; \\
0.1 \mathrm{~N} \mathrm{NaOH}, 80^{\circ} \mathrm{C}, 12 \mathrm{~h}\end{array}$ & $\begin{array}{l}\text { Deamination, } \\
\text { Ketonization }\end{array}$ & [134] \\
\hline Olanzapine & $\begin{array}{l}0.1 \mathrm{~N} \mathrm{HCl}, 60^{\circ} \mathrm{C}, 10 \mathrm{~m} ; \\
0.5 \mathrm{~N} \mathrm{NaOH}, 60^{\circ} \mathrm{C}, 30 \mathrm{~m} ; \\
\mathrm{H}_{2} \mathrm{O}, 60^{\circ} \mathrm{C}, 1 \mathrm{~h}\end{array}$ & Ketonization & [135] \\
\hline Paliperidone & $\begin{array}{l}0.2 \mathrm{~N} \mathrm{HCl}, 80^{\circ} \mathrm{C}, 24 \mathrm{~h} ; \\
0.2 \mathrm{~N} \mathrm{NaOH}, 80^{\circ} \mathrm{C}, 24 \mathrm{~h}\end{array}$ & Deamination & [136] \\
\hline Pramipexole & $\begin{array}{l}3 \mathrm{M} \mathrm{HCl}, 80^{\circ} \mathrm{C}, 48 \mathrm{~h} ; \\
2 \mathrm{M} \mathrm{NaOH}, 80^{\circ} \mathrm{C}, 24 \mathrm{~h}\end{array}$ & Deamination & [137] \\
\hline Quinacrine & $\begin{array}{l}1 \mathrm{M} \mathrm{HCl}, 80^{\circ} \mathrm{C}, 2.5 \mathrm{~h} ; \\
1 \mathrm{M} \mathrm{NaOH}, 80^{\circ} \mathrm{C}, 2.5 \mathrm{~h} ; \\
\mathrm{H}_{2} \mathrm{O}, 80^{\circ} \mathrm{C}, 4 \mathrm{~h}\end{array}$ & $\begin{array}{l}\text { Deamination, } \\
\text { Ketonization }\end{array}$ & [112] \\
\hline Ritonavir & $1 \mathrm{~N} \mathrm{HCl}, 75^{\circ} \mathrm{C}, 24 \mathrm{~h}$ & Deamination & [72] \\
\hline Sulfadimethoxine & $\begin{array}{l}0.1 \mathrm{M} \mathrm{HCl}, 80^{\circ} \mathrm{C}, 3 \mathrm{~h} ; \\
0.1 \mathrm{M} \mathrm{NaOH}, 80^{\circ} \mathrm{C}, 3 \mathrm{~h}\end{array}$ & $\begin{array}{l}\text { Deamination } \\
\text { Substitution }\end{array}$ & [76] \\
\hline Tamsulosin & $\mathrm{H}_{2} \mathrm{O}, 80^{\circ} \mathrm{C}, 48 \mathrm{~h}$ & Deamination & [138] \\
\hline Valganciclovir & $0.1 \mathrm{~N} \mathrm{HCl}, 80^{\circ} \mathrm{C}, 8 \mathrm{~h}$ & Deamination & [139] \\
\hline Zidovudine & $0.1 \mathrm{M} \mathrm{HCl}$, reflux, 5 days & Deamination & [140] \\
\hline
\end{tabular}

Table 5: Drugs degrading through dehydration assisted cyclization

\begin{tabular}{|l|l|c|}
\hline \multicolumn{1}{|c|}{ Drug } & \multicolumn{1}{|c|}{ Hydrolytic condition(s) } & Reference \\
\hline Darunavir & $0.1 \mathrm{~N} \mathrm{NaOH}, 75^{\circ} \mathrm{C}, 48 \mathrm{~h}$ & {$[36]$} \\
\hline Diclofenac & $1 \mathrm{~N} \mathrm{HCl}$, reflux, $2 \mathrm{~h}$ & {$[141]$} \\
\hline Ezetimibe & $0.1 \mathrm{M} \mathrm{NaOH}, 80^{\circ} \mathrm{C}, 8 \mathrm{~h}$ & {$[142]$} \\
\hline Enalapril & $1 \mathrm{M} \mathrm{NaOH}, 60^{\circ} \mathrm{C}, 60 \mathrm{~m} / 1 \mathrm{M} \mathrm{NaOH}, 100^{\circ} \mathrm{C}, 10 \mathrm{~m}$ & {$[42]$} \\
& $0.1 \mathrm{~N} \mathrm{HCl}, 80^{\circ} \mathrm{C}, 18 \mathrm{~h} ; \mathrm{H}_{2} \mathrm{O}, 80^{\circ} \mathrm{C}, 2$ days & {$[43]$} \\
\hline Flupirtine & $1 \mathrm{M} \mathrm{HCl}, \mathrm{RT}, 24 \mathrm{~h}$ & {$[51]$} \\
\hline Indinavir & $0.1 \mathrm{~N} \mathrm{HCl}, 80^{\circ} \mathrm{C}, 24 \mathrm{~h} ; \mathrm{H}_{2} \mathrm{O}, 80^{\circ} \mathrm{C}, 72 \mathrm{~h}$ & {$[53]$} \\
\hline Quinapril & $0.1 \mathrm{~N} \mathrm{HCl}, 80^{\circ} \mathrm{C}, 24 \mathrm{~h} ; \mathrm{H}_{2} \mathrm{O}, 80^{\circ} \mathrm{C}, 24 \mathrm{~h}$ & {$[71]$} \\
\hline Ritonavir & $0.1 \mathrm{M} \mathrm{NaOH}, \mathrm{RT}, 72 \mathrm{~h} ; \mathrm{H}_{2} \mathrm{O}, 75^{\circ} \mathrm{C}, 30 \mathrm{~h}$ & {$[72]$} \\
\hline Tamsulosin & $2 \mathrm{~N} \mathrm{NaOH}, 80^{\circ} \mathrm{C}, 72 \mathrm{~h}$ & {$[138]$} \\
\hline Tenatoprazole & $0.01 \mathrm{M} \mathrm{HCl}, \mathrm{RT}, 30 \mathrm{~m}$ & {$[143]$} \\
\hline
\end{tabular}


product (a linear conjugated anhydride) in both acidic and alkaline media ${ }^{117}$ may be explained as shown in Figure 6 . The conventional hydrolysis of amide linkage in idrocilamide yields 3-phenylacrylic acid (3-PAA) and ethanolamine. In alkaline medium, 3-PAA exists as carboxylate ion whose nucleophilic oxygen can easily displace ethanolamine from the drug to form the dimer product. In acidic medium, generation of this product may be possible by an altogether different route as outlined. Olmesartan ${ }^{64}$ is also reported to form a dimer product in acidic medium, which is an anhydride (Figure 6), similarly as in idrocilamide. The mechanism of formation of this product may be same as proposed for idrocilamde.

$\mathbf{N}$-alkylation: Amines are well known to react with alkyl halides to form the amines of higher class. It occurs through interaction between the lone pair of nitrogen atom (nucleophile) and the electron deficient $\alpha$-carbon (electrophile) of alkyl halide. ${ }^{105}$ The $\alpha$-carbon in an alcohol is significantly weak electrophile than that of an alkyl halide due to inherent difference between electronegativities of - $\mathrm{OH}$ and halide groups. However, despite this weak electrophilicity, an alcohol may also cause alkylation of amino nitrogen in a drug, if reaction conditions are conducive. Esomeprazole is such an example, in which methylation occurs at nitrogen atom of benzimidazole nucleus of the drug under acidic hydrolytic condition. ${ }^{109}$ This $\mathrm{N}$-methylation can be attributed to methanol, which is used as a co-solvent during forced degradation study. Here, the intra-molecular H-bonding may trigger a series of electron movements that causes a nucleophilic attack of benzimidazole nitrogen at methyl group of methanol (Figure 7). The released $\mathrm{OH}^{-}$ion, being a poor leaving group, immediately accepts a proton from the cationic intermediate to form $\mathrm{H}_{2} \mathrm{O}$. Candesartan cilexetil is another example, in which ethylation occurs at nitrogen atom of tetrazole ring under acidic and basic hydrolytic conditions. ${ }^{118}$ Formation of this product may be justified by a mechanism that involves a nucleophilic attack of tetrazole nitrogen at methylene group of ethanol, which is generated by hydrolytic cleavage of ether linkage on benzimidazole ring (Figure 7).

C-C bond cleavage: Cleavage of $\mathrm{C}-\mathrm{C}$ bond is very improbable under hydrolytic conditions. Fesoterodine in acidic medium ${ }^{49}$ and voriconazole in alkaline medium $^{119}$ are reported to degrade through this unusual reaction. Degradation of voriconazole due to $\mathrm{C}-\mathrm{C}$ bond cleavage may be mechanized as outlined in Figure 8. In alkaline medium, benzylic hydroxyl group is ionized to an oxide that subsequently causes cleavage of $\mathrm{C}-\mathrm{C}$ bond connecting phenyl and pyrimidine rings. This cleavage seems possible due to the pyrimidine ring that facilitates delocalization of electrons through resonance.

Dehalogenation: Aryl halides are conventionally converted into substituted product by strong nucleophile or reduced product by reducing agents in photochemical conditions. ${ }^{105}$ In gross exception to this, some drugs are reported to degrade to a substituted product, e.g. florfenicol ${ }^{50}$ and ornidazole, ${ }^{120}$ or to dehalogenated product, e.g. amlodipine ${ }^{22}$ and atorvastatin ${ }^{121}$ in acidic and/or alkaline hydrolytic condition (Figure 9). Justification for formation of such degradation products by dehalogenation reactions under hydrolytic conditions seems far away from the reality.

Hydroxylation: Alkanes and cycloalkanes are readily oxidized to alcohols by aqueous $\mathrm{H}_{2} \mathrm{O}_{2}$ in trifluoroacetic acid. ${ }^{105}$ But hydroxylation of cycloalkanes under acidic as well as alkaline hydrolytic conditions as seen in case of dexamethasone ${ }^{107}$ and simvastatin ${ }^{122}$ is quite unusual reaction. A possible mechanism for this reaction of dexamethasone in acidic medium can be explained through involvement of keto-enol tautomerization, which attracts nucleophilic addition of $\mathrm{OH}^{-}$at C-6 and subsequently displaces the hydroxyl proton at C-3 (Figure 10). Formation of this product in alkaline hydrolytic condition may be possible only through radical mediated mechanisms. In case of simvastatin, hydroxylation simply involves replacement of ethylenic hydrogens with $\mathrm{OH}^{-}$ions in alkaline medium (Figure 10).

Dehydration of carboxylic acids: A carboxylic acid readily undergoes dehydration under pyrolytic condition to form a ketene, which is stated to be an extremely reactive and unimportant functional group. ${ }^{105}$ It reacts as "super anhydride", and gives carboxylic acid with water and an ester with an alcohol. This behaviour reflects that in aqueous medium, ketene is extremely less probable to exist in free form. Rather, it may exist in equilibrium with carboxylic acid.

Oliveira et al. have reported lumiracoxib to degrade to a ketene (II) due to dehydration of $-\mathrm{COOH}$ group in acidic hydrolytic condition (Figure 11). ${ }^{123}$ However, existance of II is in gross disagreement with the exceptionally high reactive nature of ketene. Most likely, II formed due to dehydration can be converted back into lumiracoxib through hydration of the $\mathrm{C}=\mathrm{O}$ bond $\mathrm{b}^{104}$ followed by tautomerism (Figure 11). Hence, it is possible that lumiracoxib might degrade through some other mechanism to form a stable product having a mass equal to that of II. Lumaricoxib molecule contains a dihalogenated anilino group. Presence of a fluorine and a chlorine (both highly electron withdrawing) at ortho positions makes the anilinic hydrogen highly acidic, which can facilitate intra-molecular $\mathrm{H}$-transfer to generate 


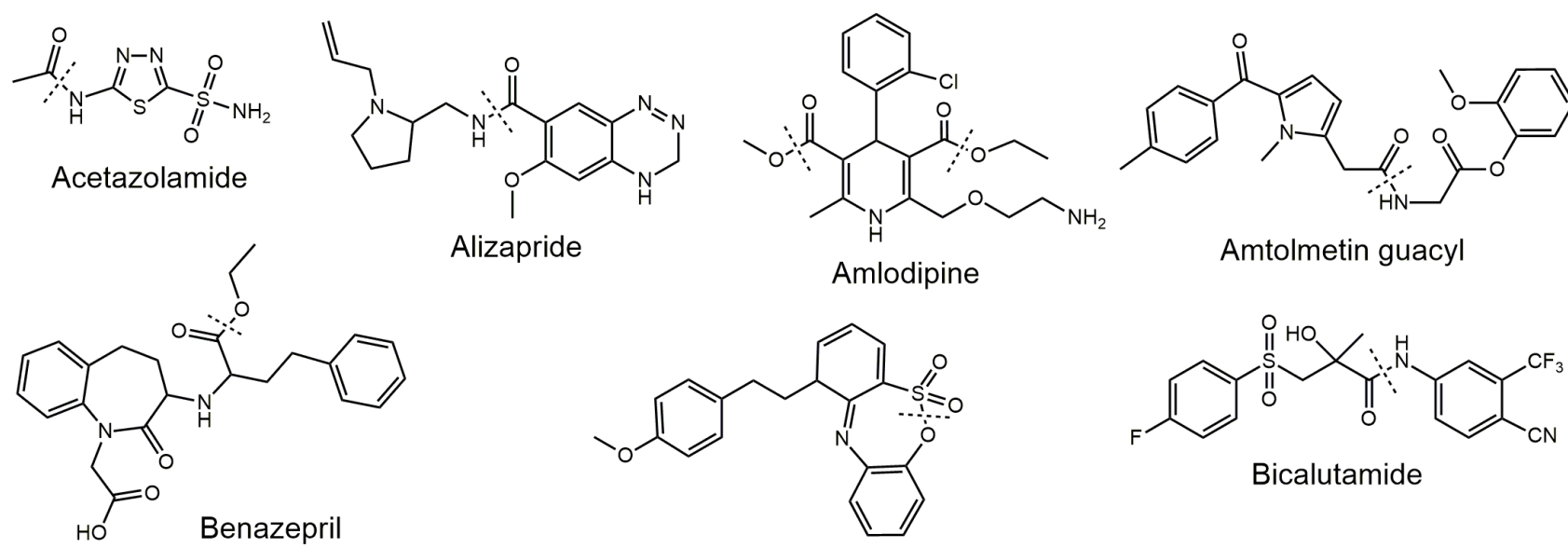<smiles>COc1ccccc1Oc1c(NS(=O)(=O)c2ccc(C(C)(C)C)cc2)nc(-c2ncccn2)nc1OCCO</smiles><smiles></smiles><smiles>NC(=O)OCC(O)c1ccccc1Cl</smiles>

Carisbamate<smiles>NC(=O)C(c1ccccc1)(c1ccccc1)C1CCC(CCc2ccc3c(c2)CCC3)C1</smiles><smiles>COC(=O)c1cc(OC)c2c(c1-c1c(C(=O)OC)cc(OC)c3c1OCO3)OCO2</smiles>

DDB<smiles>CCOC(=O)C(CCc1ccccc1)NC(C)C(=O)N1CCC[C@H]1C(=O)O</smiles>

Enalapril

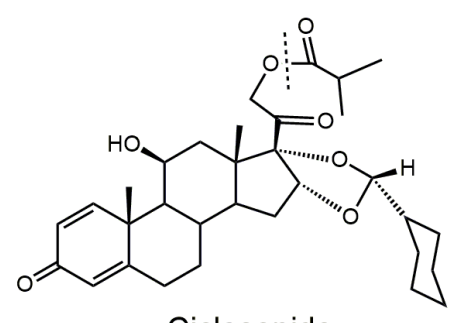

Ciclesonide<smiles>CC(C)CNCC(C)C(Cc1ccccc1)NC(=O)[O+]C1COC(O)C1</smiles>

Docetaxel

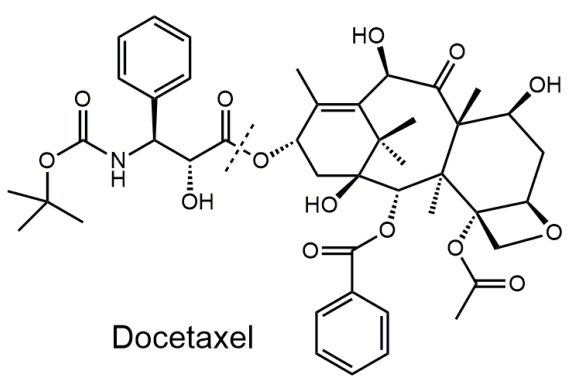<smiles>Cc1ccc(C2(O)NC(=O)c3ccccc32)cc1S(N)(=O)=O</smiles>

Chlorthalidone<smiles>COC(=O)C(c1ccccc1Cl)N1CCc2sccc2C1</smiles>

Clopidogrel<smiles>Cn1c(=O)c2c(ncn2CC2OCCO2)n(C)[13c]1=O</smiles>

Doxofylline

arunavir<smiles>Nc1ccc(O)cc1</smiles><smiles>Cc1ccc(C(F)(F)F)c(NC(=O)C2CCC3C4CCC5NC(=O)C=CC5(C)C4CCC23C)c1</smiles>

Dutasteride<smiles>CC1(C#CC2CC2)OC(=O)Nc2ccc(Cl)cc21</smiles>

Efavirenz

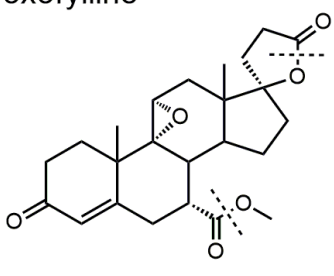

Eplerenone<smiles>CC(=O)OC1Cc2ccccc2N(C(N)=O)c2ccccc21</smiles>

Eslicarbazepine<smiles>O=C(OCCOCCO)c1ccccc1Nc1cccc(C(F)(F)F)c1</smiles>

Etofenamate 
<smiles>CC(=O)OCC(CCn1cnc2cnc(N)nc21)COC(C)=O</smiles><smiles>CCOC(=O)OC(C)(C)C(=O)Oc1ccc(C(=O)c2ccc(Cl)cc2)cc1</smiles>

Famciclovir<smiles>CCOC(=O)Nc1ccc(NCc2ccc(F)cc2)nc1N</smiles>

Flupirtine<smiles>CC(C)(C)NC(=O)[C@H]1CN(Cc2cccnc2)CCN1CC(O)CC(Cc1ccccc1)C(=O)N[C@H]1c2ccccc2C[C@H]1O</smiles><smiles>CCOC(=O)C=Cc1ccccc1C1C(C(C)=O)=C(C)NC(C)=C1C(=O)OC(C)(C)C</smiles>

Lacidipine<smiles>CC(=O)N[C@H](Cc1ccccc1)CC(O)[C@H](Cc1ccccc1)NC(=O)COc1c(C)cccc1C</smiles>

Lopinavir

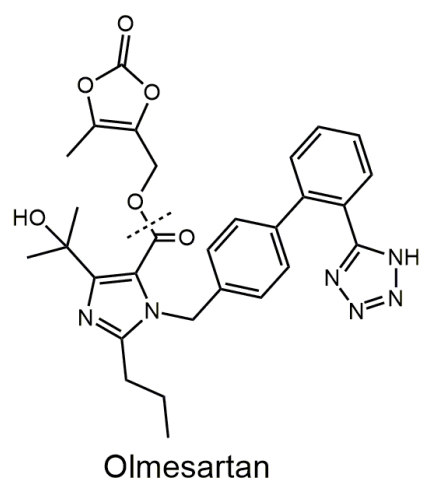

Olmesartan<smiles>NC(=O)N1c2ccccc2CC(=O)c2ccccc21</smiles>

Oxcarbazepine
Fosinipril

PAC-I<smiles>CC(C)C(=O)Oc1ccc(CO)cc1C(CCN(C(C)C)C(C)C)c1ccccc1</smiles>

Fesoterodine

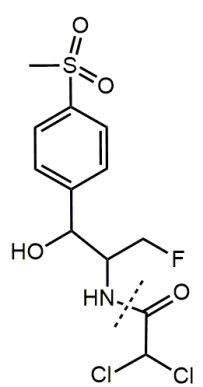

Florfenicol<smiles>CCC1=C(C)CN(C(C)(C)NCCc2ccc([Se]NC(=O)N[C@H]3CC[C@H](C)CC3)cc2)C1=O</smiles>

Glimepiride<smiles>COc1cc2c(cc1OC)CC(C)(C)CC(C)(C)C2</smiles><smiles>COCC(NC(C)=O)C(=O)NCc1ccccc1</smiles>

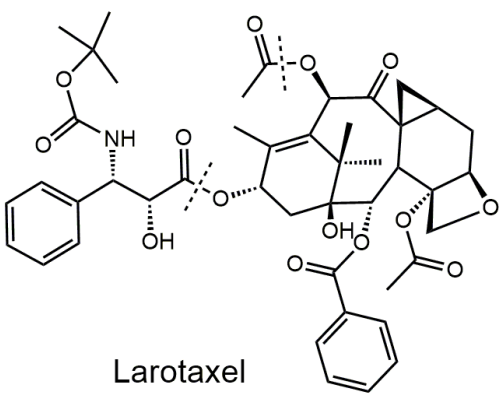
Lacosamide<smiles>CCOC(=O)N1CCC(=C2c3ccc(Cl)cc3CCc3cccnc32)CC1</smiles>
Loratadine<smiles>CN1C(C(=O)Nc2ccccn2)=C(O)c2sc(Cl)cc2S1(=O)=O</smiles>

Lornoxicam<smiles>CC(N)(N)c1ccccc1</smiles>

Niacinamide<smiles>CN1C(=O)C2C3CCC(C3)C2C(=O)N1CC1CCCCC1CN1CCN(c2nsc3ccccc23)CC1</smiles><smiles>C=CCc1cccc(/C=N/NC(C)(C)CN2CCN(Cc3ccccc3)CC2)c1O</smiles>

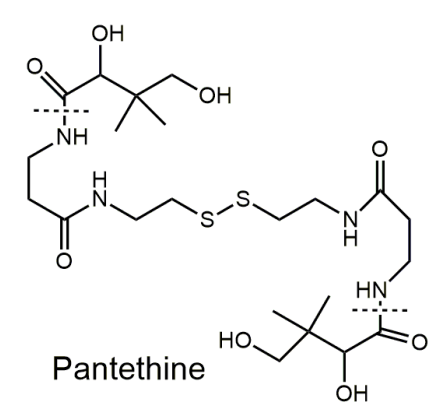


<smiles>CC(=O)Nc1ccc(O)cc1NC(C)=O</smiles>

Pentoxyfylline<smiles>CCN(CC)CCOCCOC(=O)C1(c2ccccc2)CCCC1</smiles>

Pentoxyverine<smiles>CC[NH+]1CCCC([O+]=C(C(=O)C(O)(c2ccccc2)c2ccccc2)c2ccccc2)C1</smiles>

Pipenzolate<smiles>CCOC(=O)C(CCc1ccccc1)NC(C)C(=O)N1Cc2ccccc2C1C(=O)O</smiles>

Quinapril<smiles>CC(C)c1nc(CN(C)C(=O)NC(C(=O)NC(Cc2ccccc2)CC(O)C(Cc2ccccc2)NC(=O)OCc2cncs2)C(C)C)cs1</smiles><smiles>CCN(C)C(=O)Oc1cccc(C(C)N(C)C)c1</smiles>

Rivastigmine<smiles>CCOCCNC(=O)c1ccc(Cl)s1</smiles><smiles>CCCN(CCC)CCc1cccc2c1CC(=O)N2</smiles>

Ropinirole<smiles>CCOC(=O)c1ccc(C#Cc2ccc3c(c2)C(C)(C)CCS3)nc1</smiles><smiles>COc1c(OC2OC(CO)C(O)C(O)C2O)cc2c(c1OC)-c1ccc(SC)c(=O)cc1[C@H](NC(C)=O)CC2</smiles><smiles>Cc1cccc(Nc2ccncc2S(=O)(=O)NC(=O)NC(C)C)c1</smiles>

Torasemide<smiles>CCCCC(=O)N(Cc1ccc(-c2ccccc2-c2nnn[nH]2)cc1)C(C(=O)O)C(C)C</smiles>

Valsartan<smiles>N#Cc1ccc2[nH]cc(CCCCN3CCN(c4ccc5oc(C(N)=O)cc5c4)CC3)c2c1</smiles>

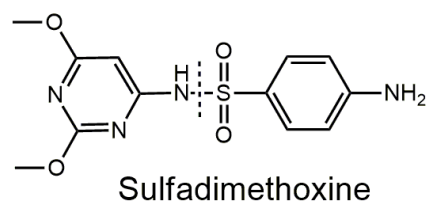

Sulfadimethoxine

\section{Thiocolchicoside}

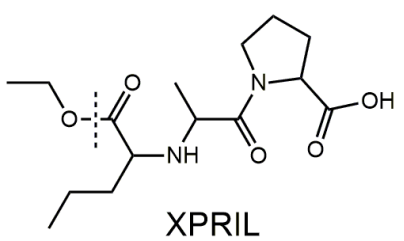

Vilazodone<smiles>C/C=C(/NC(=O)c1csc(CNC(=O)CC(/C=C\CCS(C)(=O)=O)OC(=O)C(NC(=O)C(C)=O)C(C)C)n1)C(C)C</smiles>

YK-1101<smiles>CC(CSC(=O)c1ccccc1)C(=O)[N+]1(C)CCC(Sc2ccccc2)CC1C(=O)O</smiles><smiles>Cc1ccccc1</smiles>

\section{Zolpidem}

Figure 1(a)-1(c): Some drugs known to degrade through hydrolysis of amide and ester linkages. 


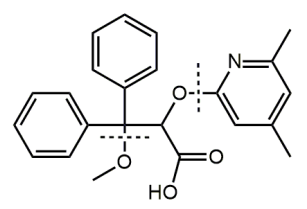

Ambrisentan<smiles>CCOc1nc2cccc(C(=O)OC(C)OC(=O)OC3CCCCC3)c2n1Cc1ccc(-c2ccccc2-c2nnn[nH]2)cc1</smiles>

Candesartan cilexetil

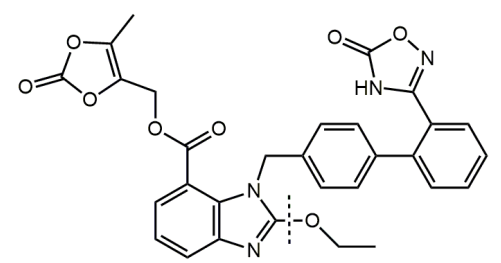

Azilsartan<smiles>COc1ccccc1Oc1c(NS(=O)(=O)c2ccc(C(C)(C)C)cc2)nc(-c2ncccn2)nc1OCCO</smiles><smiles></smiles>

Doxofylline<smiles>O=C(OC[Te]OCCO)c1ccccc1Nc1cccc(C(F)(F)F)c1</smiles>

Etofenamate<smiles>COc1cc2c(c(OC)c1OC)-c1ccc(SC)c(=O)cc1C(NC(C)=O)CC2</smiles>

Thiocolchicoside<smiles>Cn1c(=O)cc(N2CCC[C@@H](N)C2)n(Cc2ccccc2C#N)c1=O</smiles>

Alogliptin<smiles>CC(=O)[C@]1(O)Cc2c(O)c3c(c(O)c2[C@@H](OC2(C)CC(N)C(O)C(C)O2)C1)C(=O)c1ccccc1C3=O</smiles>

Idarubicin $\quad \mathrm{NH}_{2}$<smiles>CNCC[C](Oc1cccc2ccccc12)c1cccs1</smiles><smiles>CCNc1nc2ccc(Cl)cc2c(C)c1C(C)(C)c1ccccc1</smiles>

Etifoxine
Lacosamide<smiles>COCC(NC(C)=O)C(=O)NCc1ccccc1</smiles>

NPC $1161 \mathrm{C}$<smiles></smiles>

Topiramate<smiles>CN(C)CCOc1ccc(C(=C(CCCl)c2ccccc2)c2ccccc2)cc1</smiles>

Toremifene<smiles>CN(C)CCCC1(c2ccc(F)cc2)OCc2cc(C#N)ccc21</smiles>

Citalopram

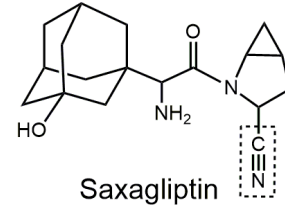<smiles>N#Cc1ccc2[nH]cc(CCCCN3CCN(c4ccc5oc(C(N)=O)cc5c4)CC3)c2c1</smiles><smiles>CCN(C(C)=O)c1cccc(-c2ccnc3c(C#N)ccn23)c1</smiles>

Zaleplon<smiles></smiles>

Pridinol

Figure 2: Drugs degrading through hydrolysis of ether, nitrile and N-nitrosoamine groups. 


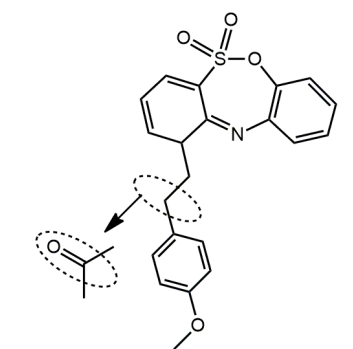

Benzopyridooxathiazepine

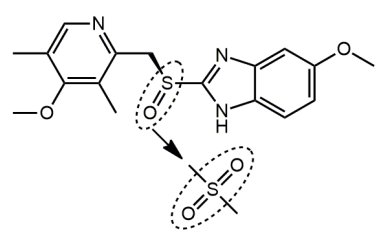

Esomeprazole

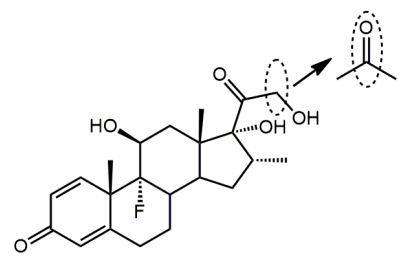

Dexamethasone

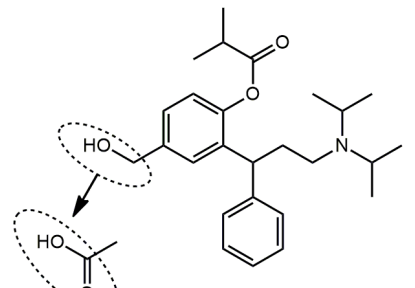

Fesoterodine
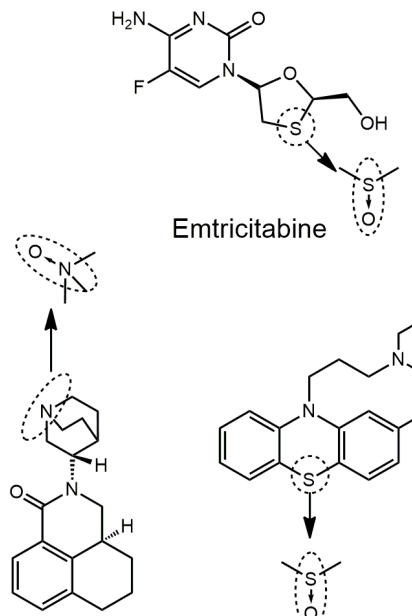

Palonosetron

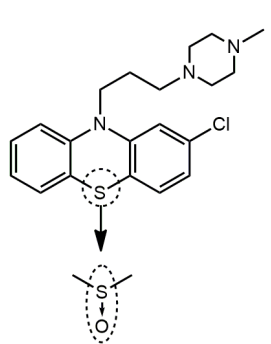

Perchlorperazine

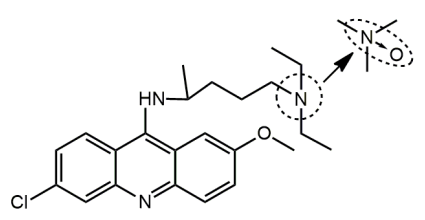

Quinacrine
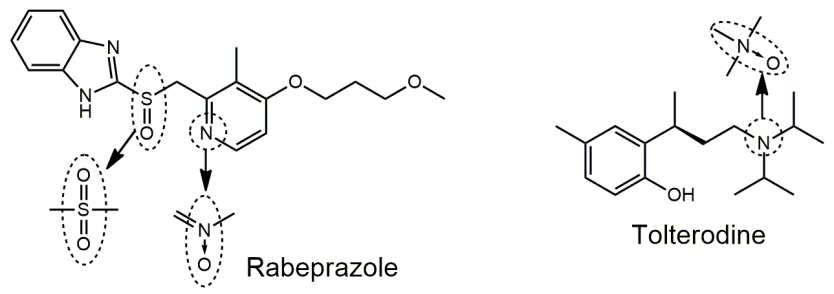

Figure 3: Drugs degrading through oxidation under hydrolytic conditions. The chemical change is marked by dotted ovals and arrows<smiles>CNCCC(Oc1cccc2ccccc12)c1cccs1</smiles>

Duloxetine<smiles>COc1ccccc1Oc1c(NS(=O)(=O)c2ccc(C(C)(C)C)cc2)nc(-c2ncccn2)nc1OCCO</smiles><smiles>CC(C)O</smiles>

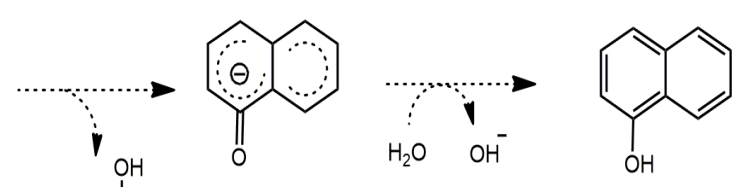

1-Naphthol<smiles>CNCCC(O)c1cccs1</smiles>

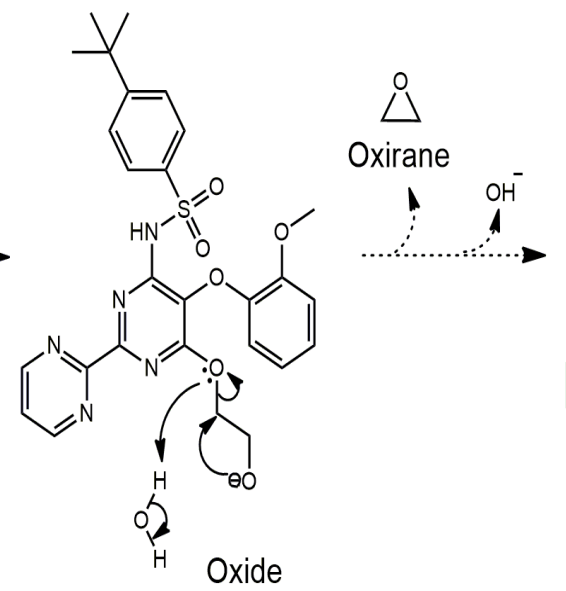<smiles>COc1ccccc1Oc1c(O)nc(-c2ncccn2)nc1NS(=O)(=O)c1ccc(C(C)(C)C)cc1</smiles>

Figure 4: Proposed mechanisms of hydrolysis of duloxetine and bosentan in alkaline medium. 

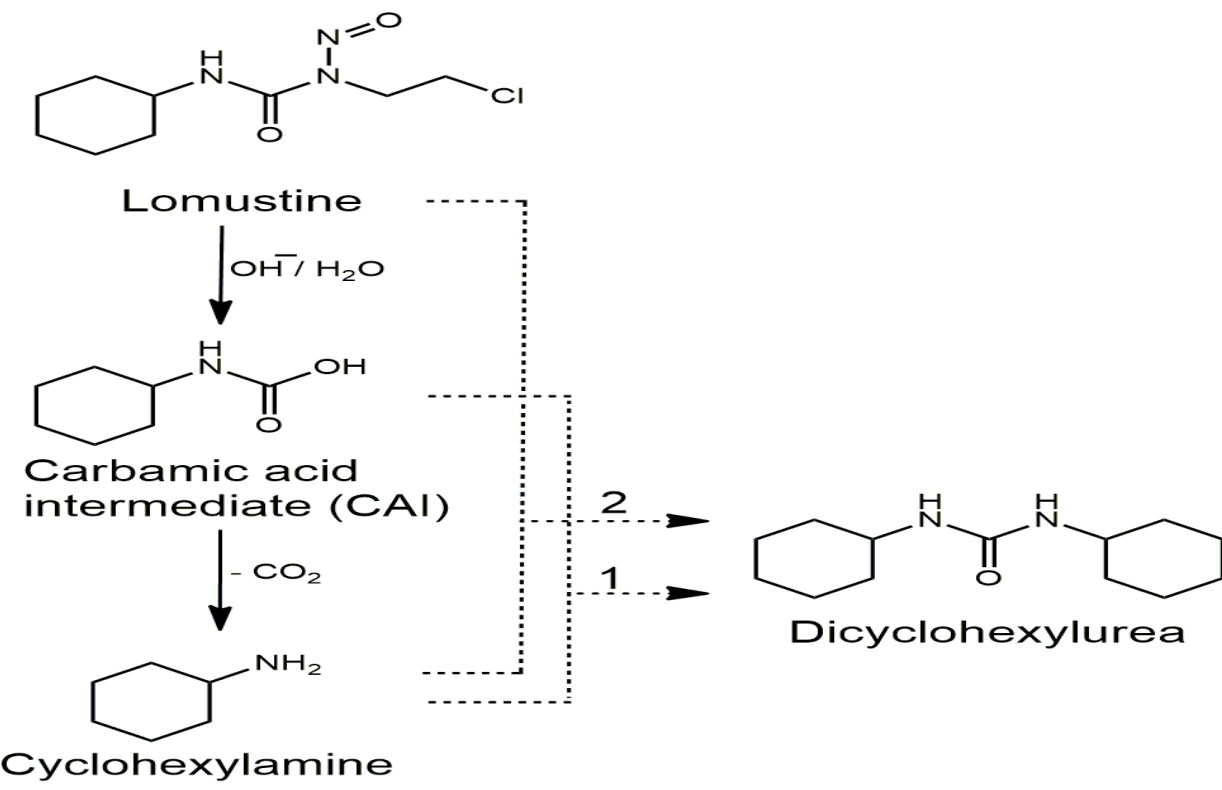

Figure 5: Proposed mechanism of hydrolytic degradation of lomustine to dimeric product.

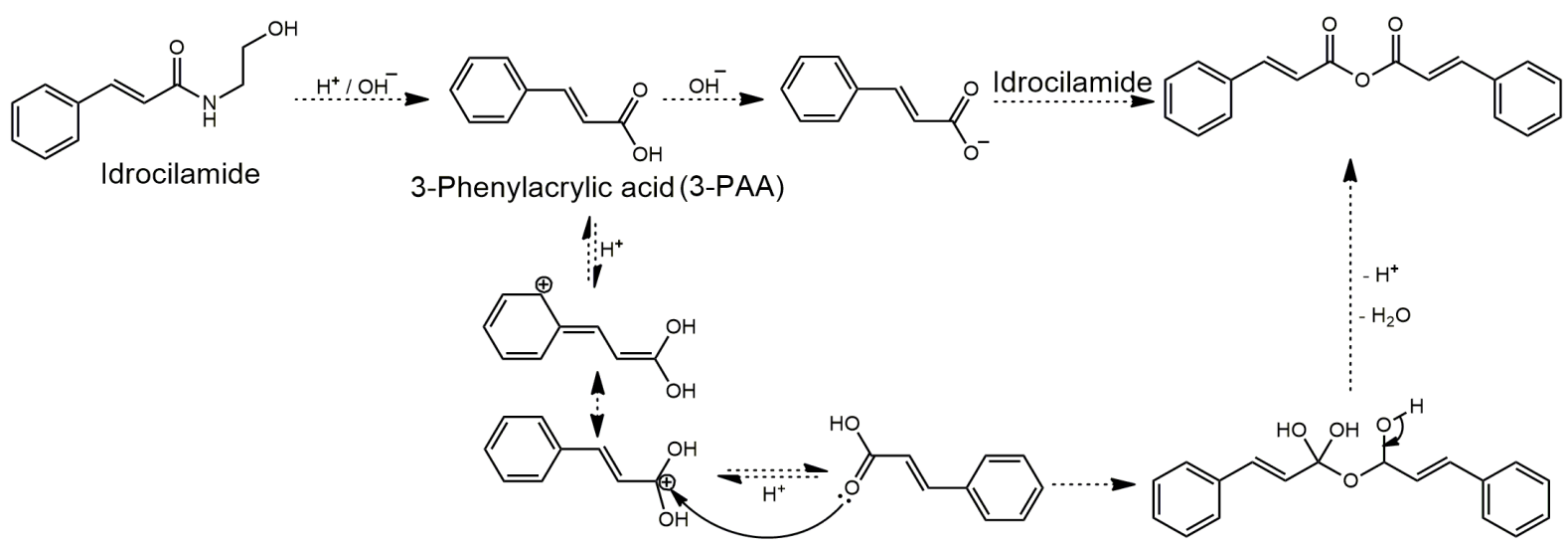

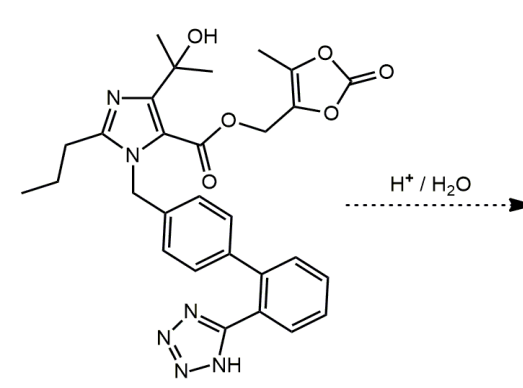

Olmesartan

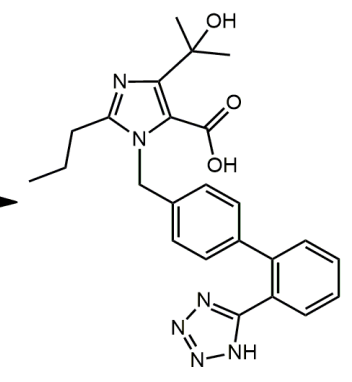

(I)

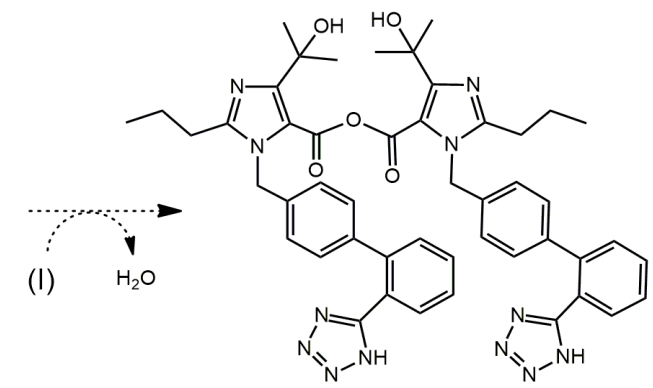

Figure 6: Proposed mechanisms of hydrolytic degradation of idrocilamide and olmesartan to dimer products. 

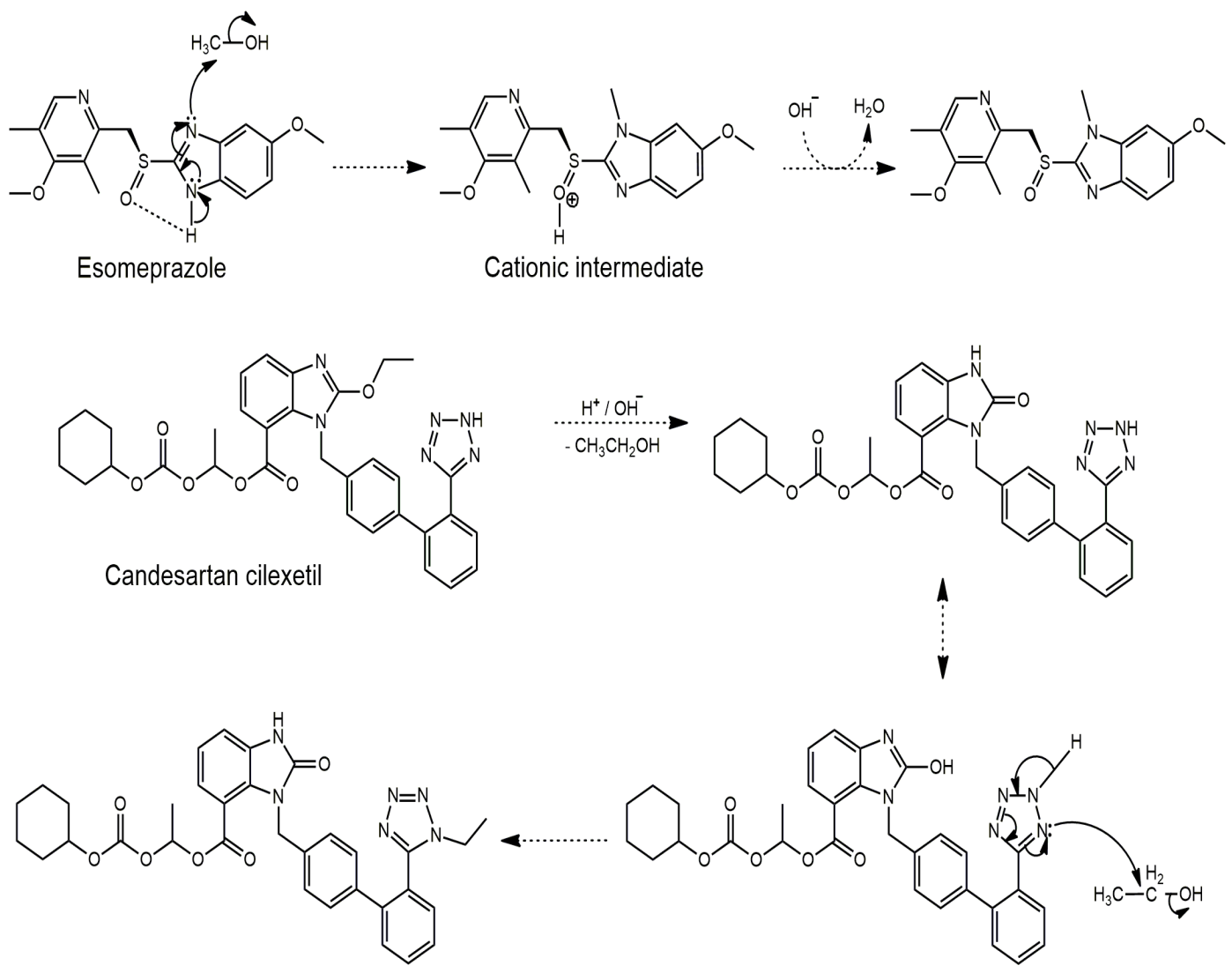

Figure 7: Proposed mechanisms of N-Alkylation of esomeprazole and candesartan cilexetil.<smiles>CC(c1ncncc1F)C(C)(Cn1cncn1)c1ccc(F)cc1F</smiles>

Voriconazole

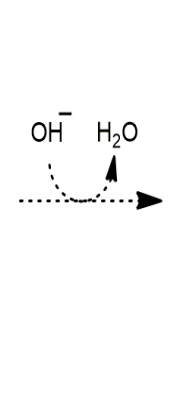

Figure 8: Proposed mechanism of deallylation of voriconazole.

a nitranion (III). The nucleophilic nitrogen in III readily attacks the electrophilic carboxylic carbon and releases $\mathrm{H}_{2} \mathrm{O}$ to form a lactam product, which has mass equal to that of II (Figure 11), and is also more stable than II. The correct structure can be conveniently allotted on the basis of fragment peaks in the mass spectral data of the product.

C-N bond cleavage: Conversion of cytosine to uracil, and of guanine to xanthine are classical examples of deamination reactions ( $\mathrm{C}-\mathrm{N}$ bond cleavage) through enzymatic catalysis. ${ }^{124,125}$ However, such conversion under non-enzymatic hydrolytic environment is not well reported in standard literature. Despite it, many drugs are known to degrade to ketonic or other substituted products due to cleavage of $\mathrm{C}-\mathrm{N}$ bond in both acidic as well as alkaline medium (Table 4). Ketonic products are formed from drugs belonging to category of enamines in acidic as well as alkaline medium through mechanism proposed (Figure 12) as follows: Under acidic condition, protonation of enamine generates a quaternary amine, 


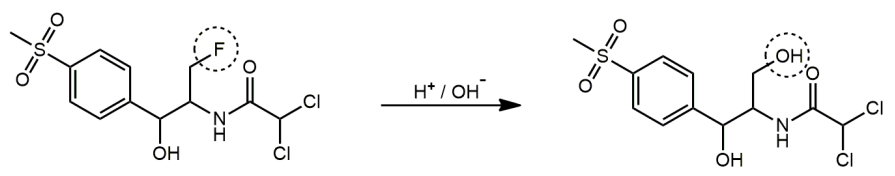

Florfenicol<smiles>Cc1ncc([N+](=O)[O-])n1CC(O)Cn1c([N+](=O)[O-])cnc1C</smiles>
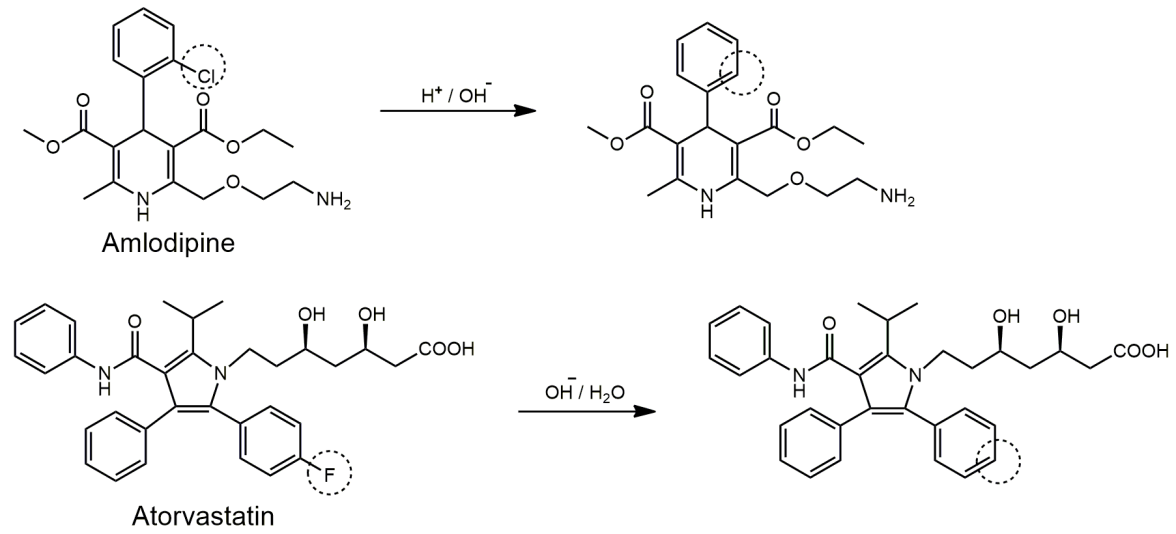

Figure 9: Dehalogenation under hydrolytic conditions.

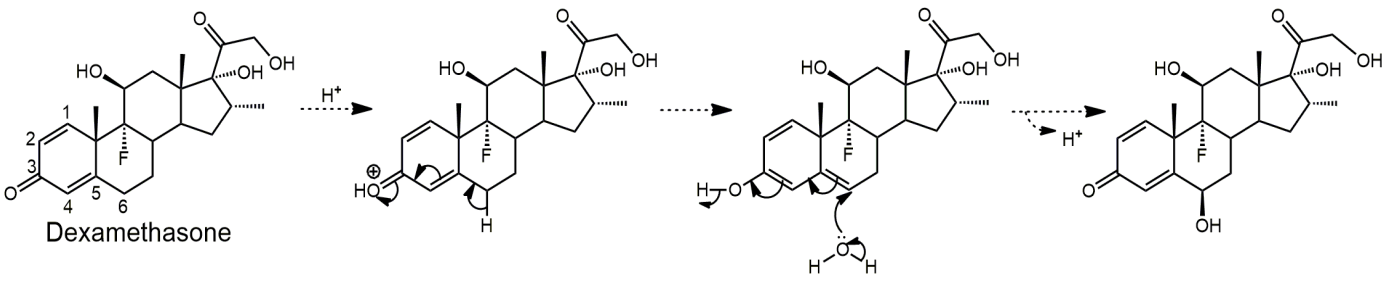<smiles>CCC(C)(C)C(=O)O[C@H]1CC(C)C=C2C=CC(C)C(CCC3CC(O)CC(=O)O3)C2C1CCC1CC(O)CC(=O)O1</smiles>

Figure 10: Acid and base catalyzed hydroxylation of dexamethasone and simvastatin, respectively.

which makes the $\alpha$-carbon an electrophilic centre. The latter attracts the nucleophilic attack by $\mathrm{H}_{2} \mathrm{O}$ to form an amino alcohol intermediate. Subsequently, under the influence of heat, $\mathrm{C}-\mathrm{N}$ bond is cleaved to form ketonic product with the liberation of an amine and free acid. Under alkaline condition, $\mathrm{OH}^{-}$ion (a strong nucleophile) attacks directly at electron deficient $\alpha$-carbon resulting in displacement of amine and formation of an enol, which is tautomerized to the ketonic product. In case of formation of substituted products, the reaction will stop at the isomerisation stage to produce unsaturated chloro product.

Drugs having saturated amine groups degrade due to partial charge separation across the $\mathrm{C}-\mathrm{N}$ bond owing to its polarity (Figure 12). In acidic medium, this charge separation is exaggerated by protonation of amino nitrogen that attracts nucleophilic attack of $\mathrm{H}_{2} \mathrm{O}$ (weak nucleophile), whereas in alkaline medium, $\mathrm{OH}^{-}$(a strong 

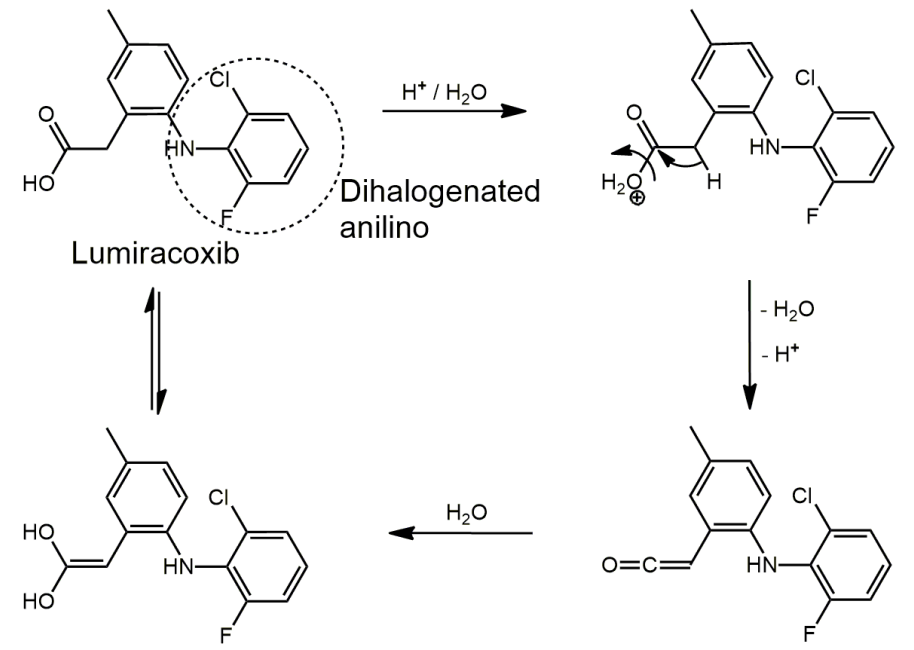

Ketene product $(\mathrm{II}$, Mass $=275.05)$

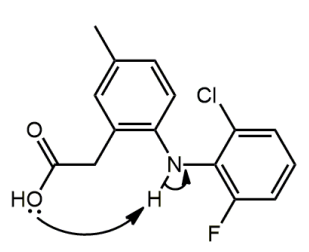

Lumiracoxib

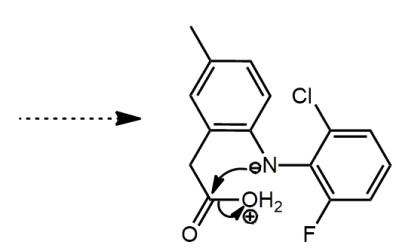

(III)

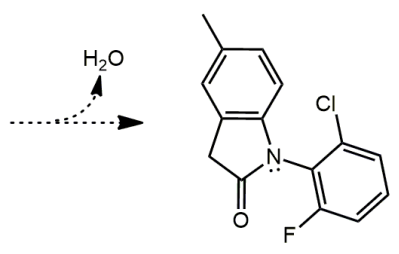

Lactam product $($ Mass $=275.05)$

Figure 11: Degradation of lumiracoxib under acidic hydrolytic condition to a ketene or lactam product.<smiles>C/C=C(\C)N(C)C</smiles><smiles>[10BH][10BH]</smiles>

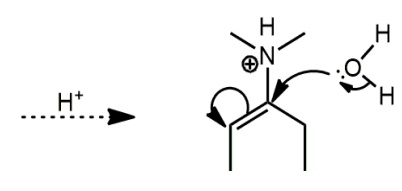

Quaternary amine<smiles>CCC(CC)(CC)C(CC)(CC)N(C)C</smiles>
Amino alcohol intermediate

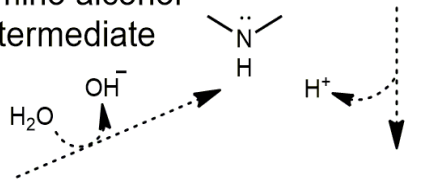<smiles>CCC1=C(C)CC(CCO)C1(CC)N(C)C</smiles>

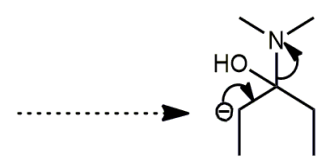<smiles>CCC(O)=C(C)C[C@@H](OCN(C)C)C(C)C</smiles>

Enol<smiles>CC#CC#CCCCC(=O)CC</smiles>

Ketonic product

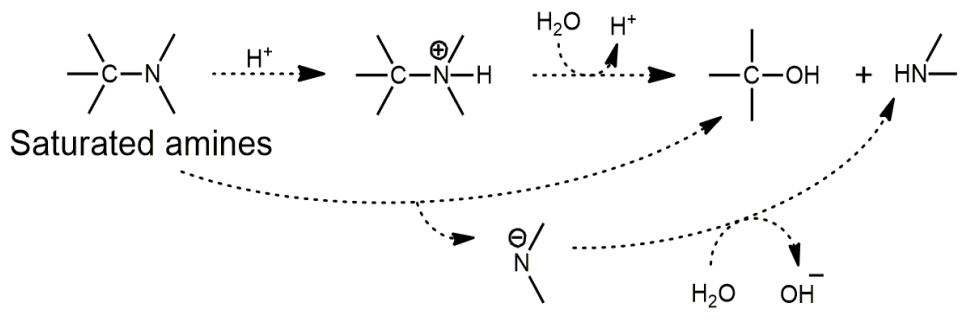

Figure 12: Proposed mechanisms of $\mathrm{C}-\mathrm{N}$ bond cleavage. 


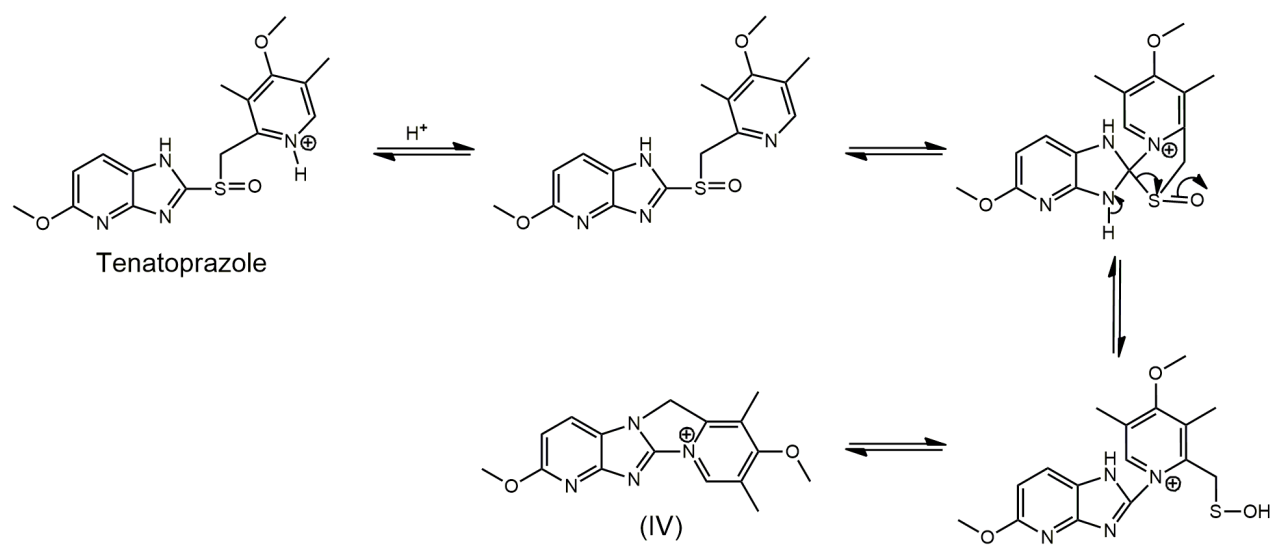

Figure 13: Formation of an indene product from tenatoprazole, which is not in agreement with textbook organic chemistry and mass spectrometry.

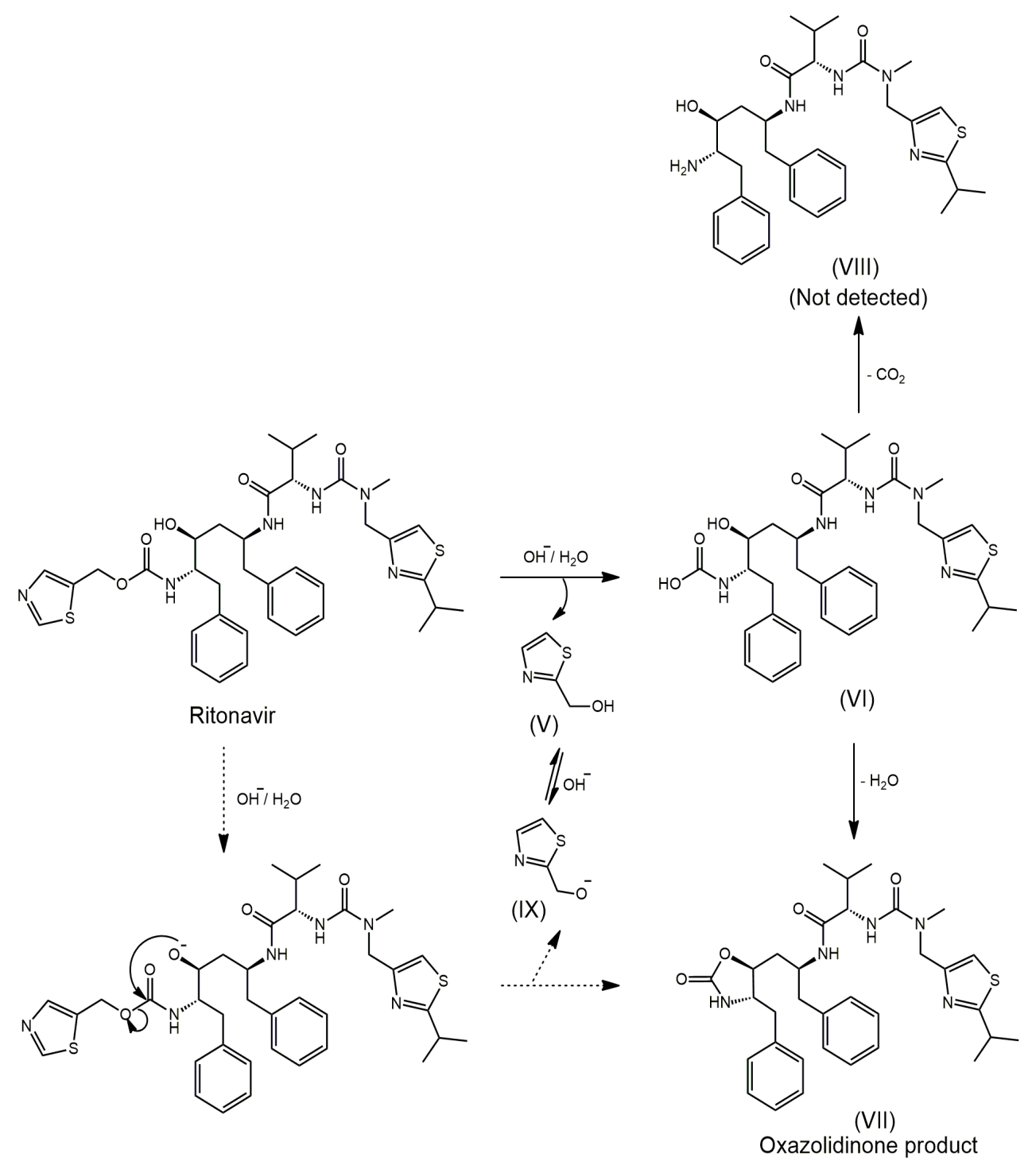

Figure 14: Reported and proposed routes of degradation of ritonavir. 

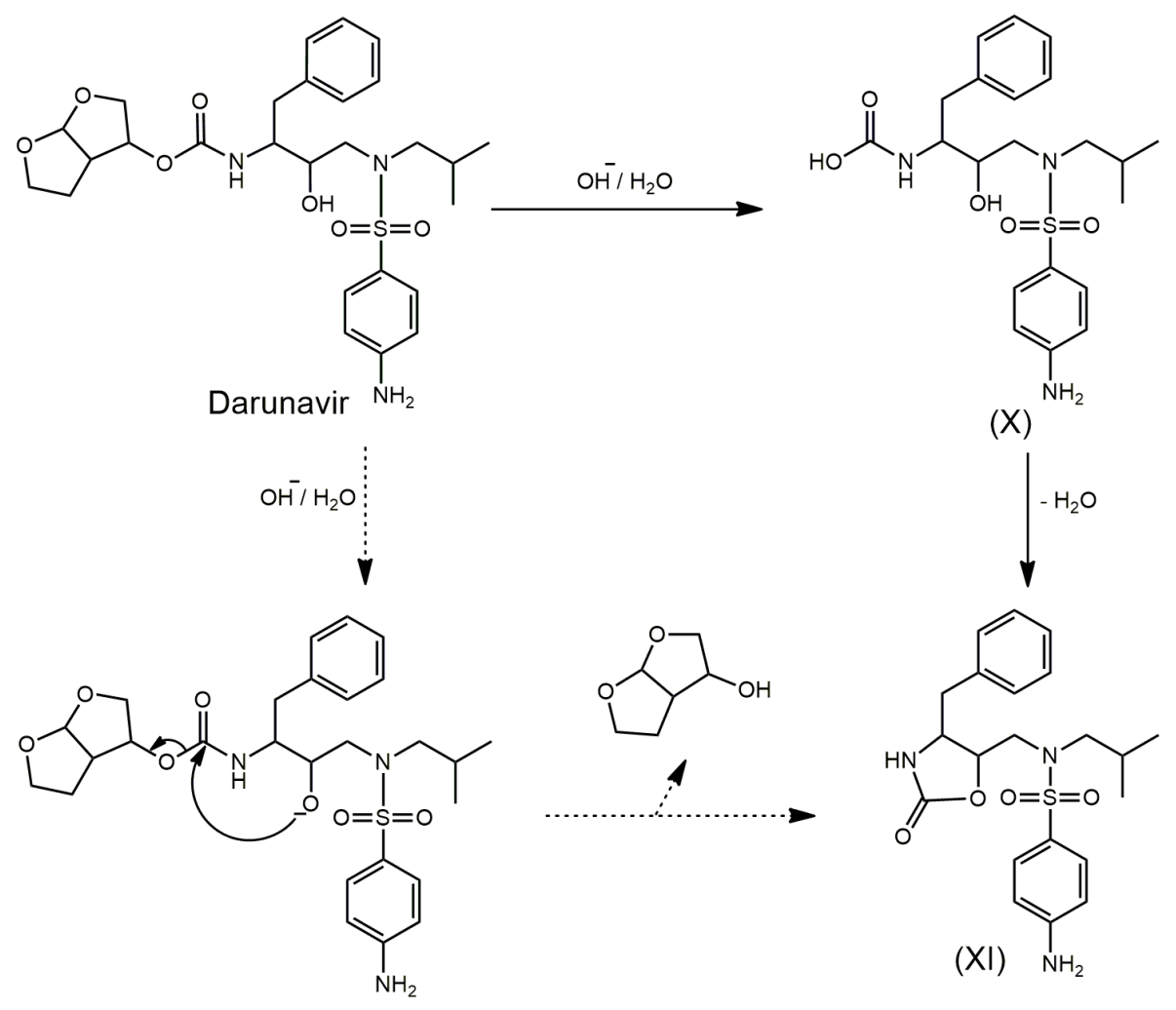

Figure 15: Reported and proposed mechanisms of degradation of darunavir to an oxazolidinone product.

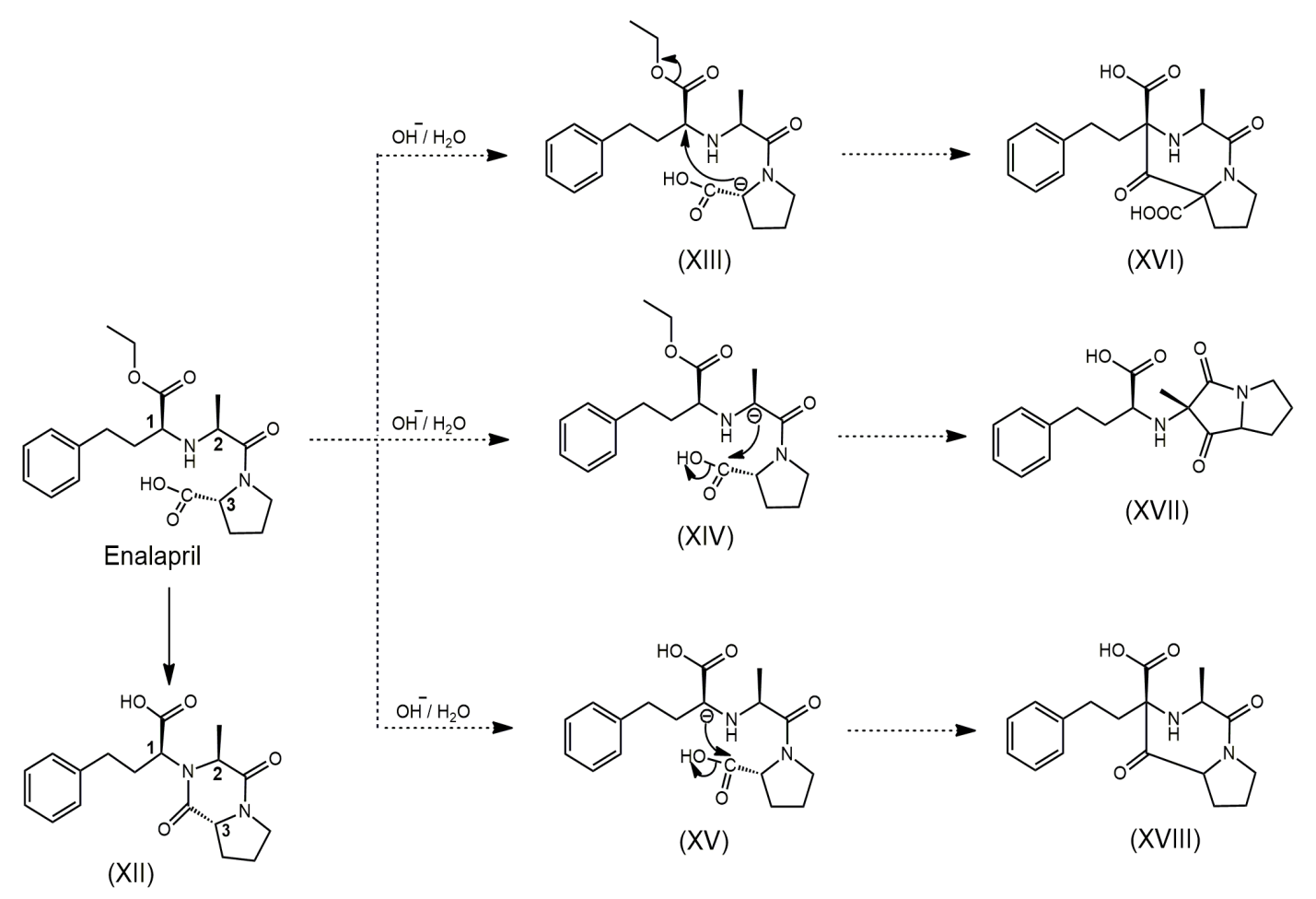

Figure 16: Possible cyclic degradation products of enalapril. 


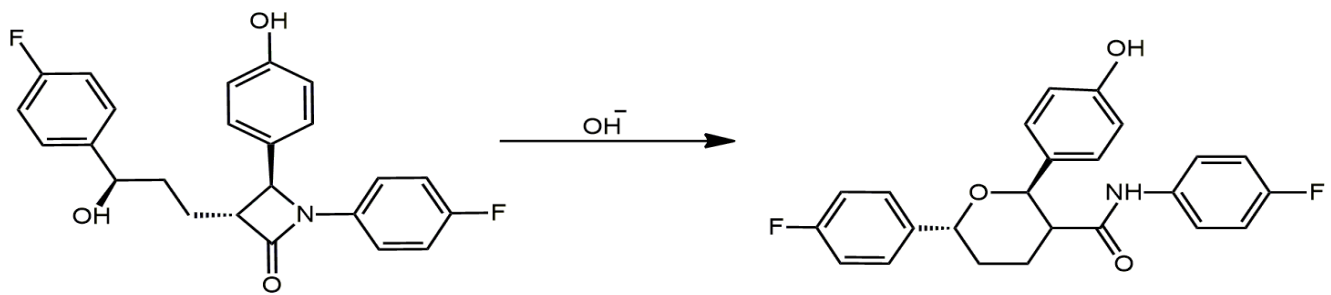

Ezetimibe<smiles>O=C(O)C(CCC(O)c1ccc(F)cc1)C(Nc1ccc(F)cc1)c1ccc(O)cc1</smiles>

$(\mathrm{XIX})$

Figure 17: Degradation of ezetimibe through ring enlargement.

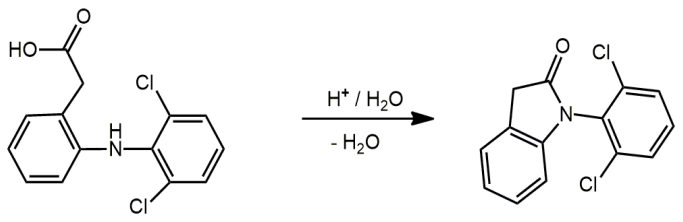

Diclofenac

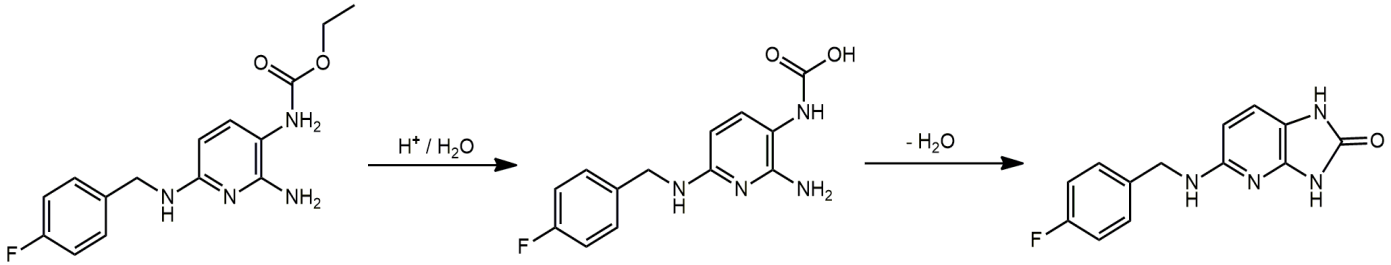

Flupirtine<smiles>CCOC(=O)C(CCc1ccccc1)NC(C(=O)O)N1Cc2ccccc2CC1C(=O)O</smiles>

Quinapril
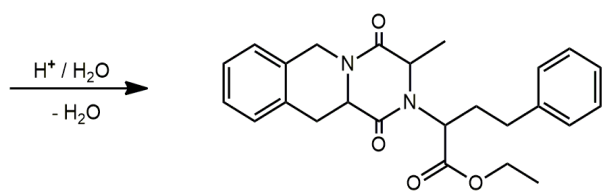

Figure 18: Mechanism of lactam ring formation in diclofenac, flupirtine and quinapril. 


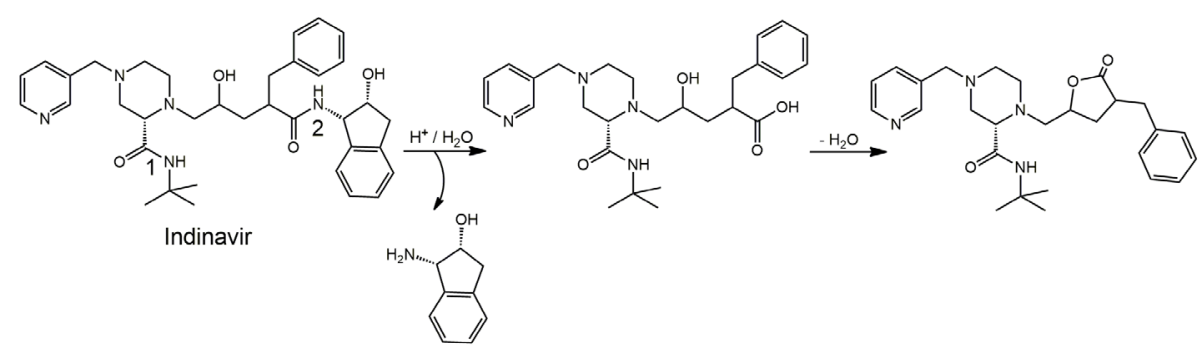

Figure 19: Mechanism of lactone ring formation in indinavir.

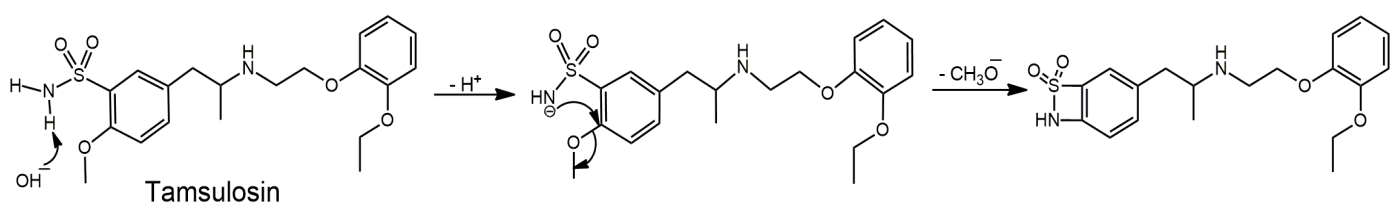

Figure 20: Mechanism of cyclization in tamsulosin.

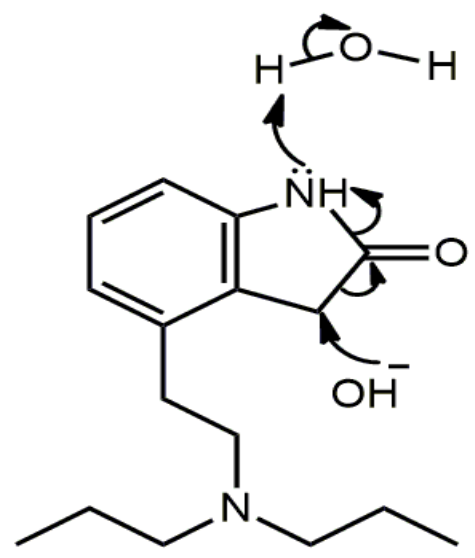

Ropinirole

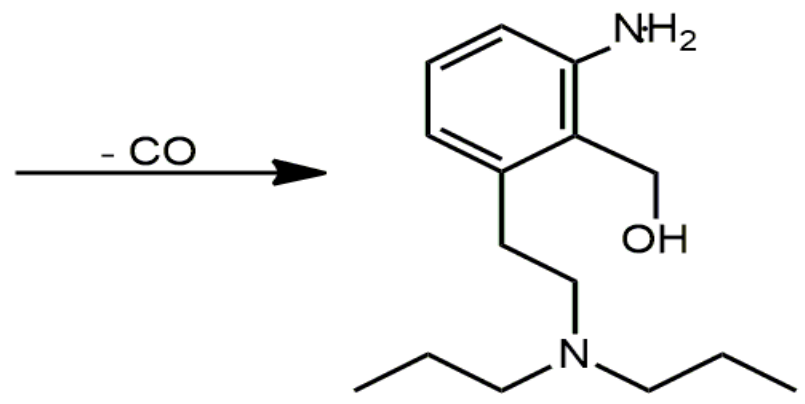

$(\mathrm{XXI})$

Figure 21: Mechanism of hydrolytic cleavage of ropinirole in alkaline medium.

nucleophile) is attracted by the electron deficient $\alpha$-carbon. The relative susceptibility of different amine drugs to $\mathrm{C}-\mathrm{N}$ bond cleavage depends largely on the nature of leaving group, which in this case is an amine. The order of strength of leaving abilities of different amines is $3^{\circ}>2^{\circ}>1^{\circ}>\mathrm{NH}_{3}>$ aromatic amines. In addition, any functional group that increases electrophilicity of $\alpha$-carbon increases the leaving ability of the amino group.

Cyclization: Under hydrolytic conditions, a C-O, C-N, C-S and C-X bond is cleaved through addition of a $\mathrm{H}_{2} \mathrm{O}$ molecule. In contrast to it, some drugs are reported to degrade under hydrolytic condition (Table 5) to cyclic products, and these products are possible to form only through dehydration assisted formation of lactam $(\mathrm{C}-\mathrm{N})$ or lactonic $(\mathrm{C}-\mathrm{O})$ linkages.

Tenatoprazole degrades to an indene derivative (IV) under both acid and alkali hydrolytic conditions. A mechanism of formation of this product in acid hydrolysis is also outlined (Figure 13), wherein it is reported that "under acidic conditions, the pyridinyl nitrogen is protonated to form pyridinium. Then the lone pair of electrons on the pyridinyl nitrogen undergoes nucleophilic attack at the electron deficient carbon (2nd carbon) of the imidazole ring. This results in the formation of indene derivative of tenatoprazole". ${ }^{143}$ A careful analysis of this outlined mechanism reveals that this proposition is self contradictory because of two observations. Firstly, protonation of pyridinyl nitrogen will use up its lone 
<smiles>Cc1ncc([N+](=O)[O-])n1CC(O)CCl</smiles>

Ornidazole<smiles>Cc1ncc(N=C2CCCC2)n1CC1CCCCC1CO</smiles>

N

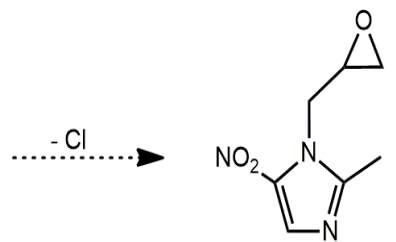

Oxirane product

Figure 22: Proposed mechanism of degradation of ornidazole
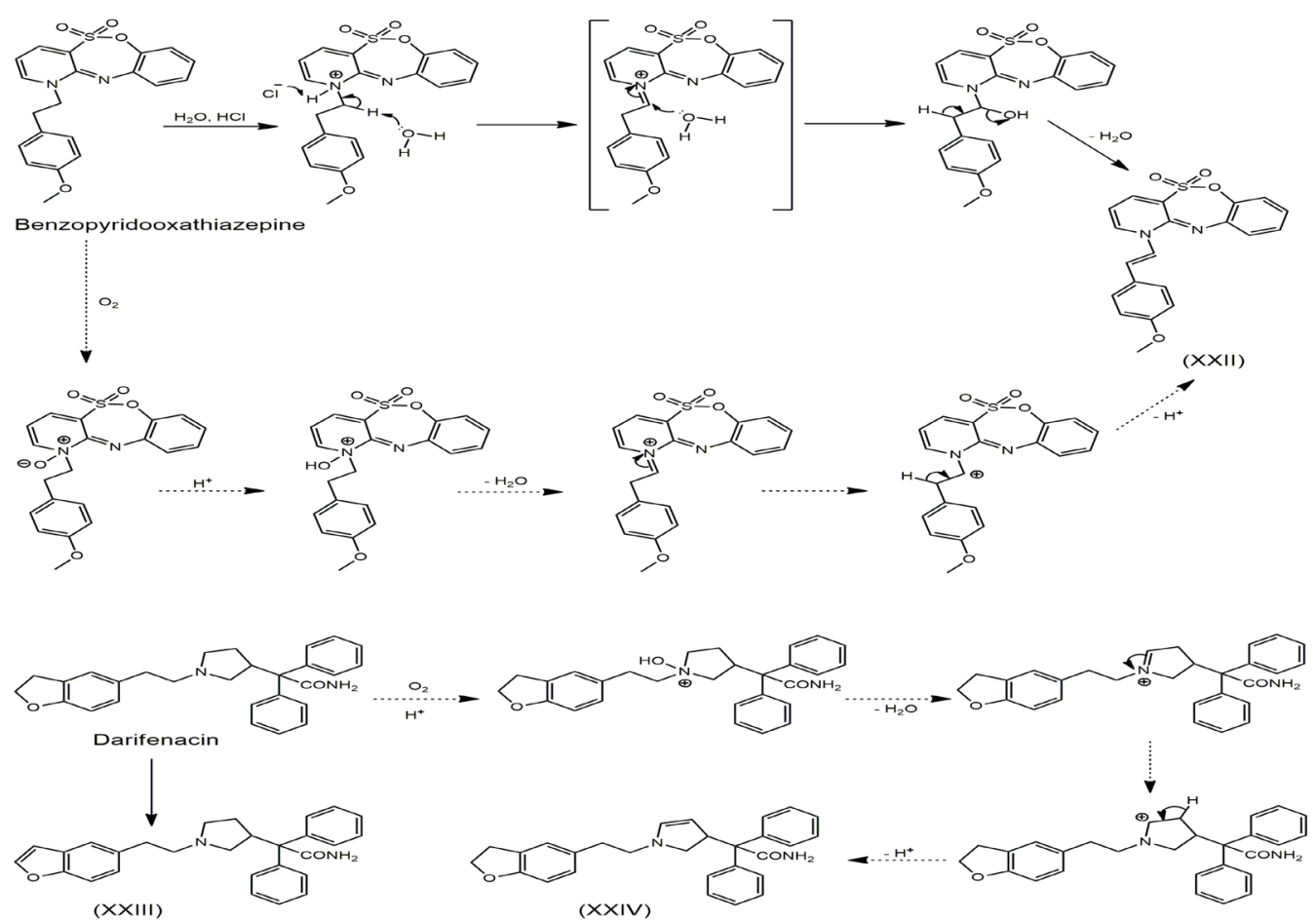

Figure 23: Mechanisms of dehydrogenation of benzopyridooxathiazepine and darifenacin<smiles>CC(O)C[C@]1(C(=O)O)CC(Sc2ccccc2)CCN1C(=O)C(C)CSC(=O)c1ccccc1</smiles>

Figure 24: Reduction of carboxylic acid in zofenopril 


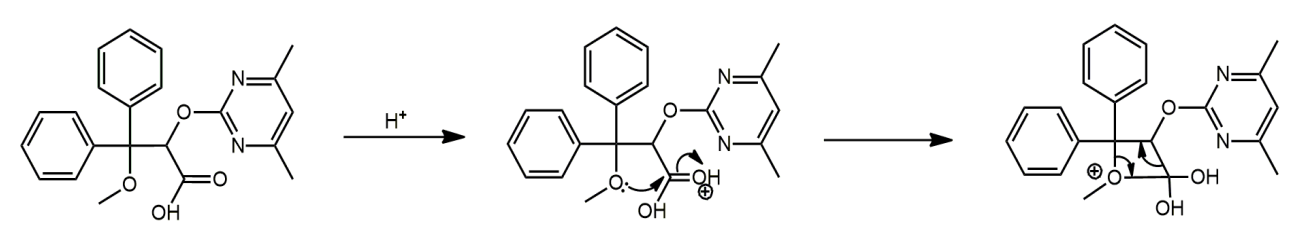

Ambrisentan
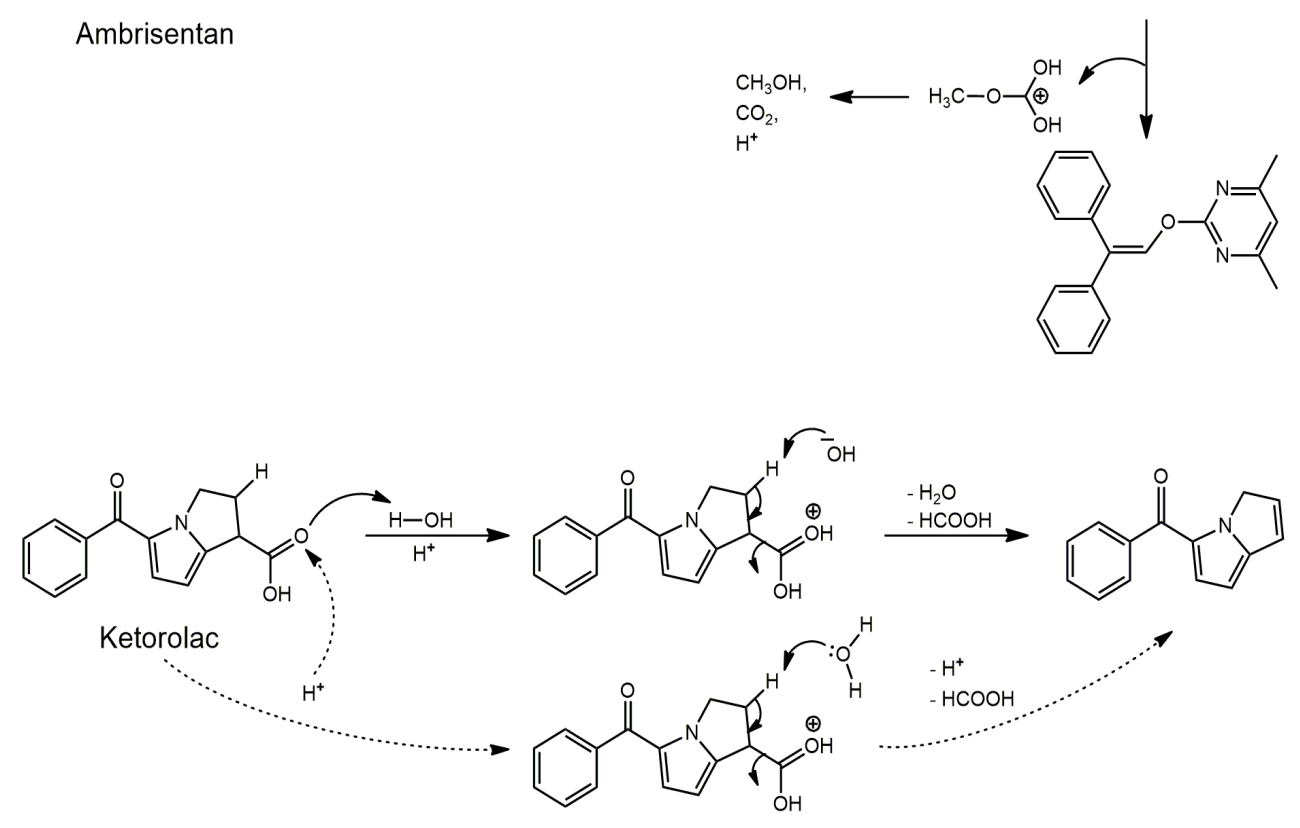

Figure 25: Decarboxylation under hydrolytic condition

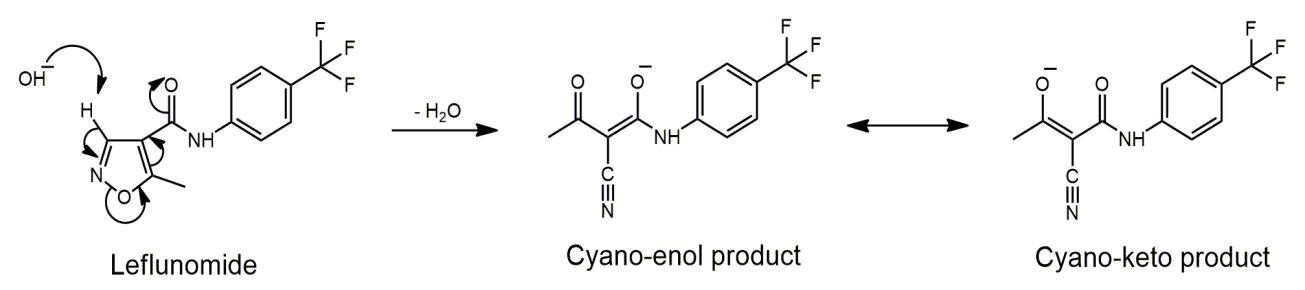

Figure 26: Mechanism of isoxazole ring opening in leflunomide
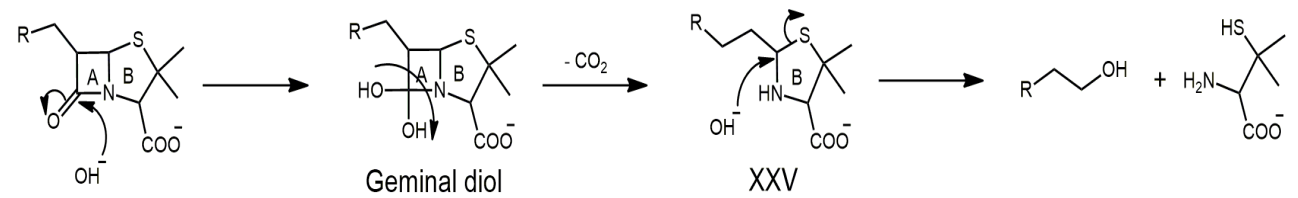

Ampicillin

Flucloxacillin

Figure 27: Mechanism of bicyclic ring opening in ampicillin and flucloxacillin

pair of electrons, and hence these are not available for nucleophilic attack on C-2 of imidazole. Secondly, the product is detected at $m / z 298$ in + ESI mode suggesting it to contain an odd number of nitrogen atoms. ${ }^{144}$ But the reported structure of the degradation product contains an even number (four) of nitrogen atoms. Based on these observations, it may be understood that struc- ture of the product as proposed in the report is not correct.

Ritonavir is reported to degrade through hydrolytic cleavage of urethane linkage to form thiazol-5-yl methanol $(\mathrm{V})$, and a carbamic acid product (VI) under alkaline hydrolytic condition. The latter subsequently loses $\mathrm{H}_{2} \mathrm{O}$ to form an oxazolidinone product (a cyclized degradation product, VII) (Figure 14). ${ }^{72}$ This route seems less 
probable given to the fact that a carbamic acid is highly unstable, which under hydrolytic conditions readily tends to lose $\mathrm{CO}_{2}$ to form an amine (VIII). Incidentally, VIII is not reported to be detected in the study. Contrary to this reported route, the same product (VIII) can be conveniently formed through another single step route that involves nucleophilic attack of alkoxy oxygen onto carbonyl carbon of urethane linkage resulting in the release of 5-oxathiazolylmethoxide (IX) as leaving group (Figure 14). On the similar note, darunavir is reported to degrade through hydrolytic cleavage of carbamate linkage to form a carboxylic acid (X) under alkaline condition that subsequently undergoes dehydration to form an oxazolidinone product (XI) ${ }^{36}$ But here again, the formation of the product can be explained by the alternate mechanism as proposed for degradation of ritonavir to product VII (Figure 15).

Toporisic et al. have reported that enalapril degrade to a piperidinone-pyrrolidine fused product (XII) at $100{ }^{\circ} \mathrm{C}$ involving the formation of an amide bond. ${ }^{42}$ However, this route of formation of XII seems to be less probable because formation of an amide bond in alkaline medium at such a high temperature is neither reported nor supported in literature. It reveals that enalapril might have degraded to some other product having mass same as that of XII. A concentrated look into structure of enalapril reveals that there are three active hydrogens, ${ }^{1-3}$ that can generate carbanions (XIII-XV, respectively) in the presence of a base (Figure 16). The carbanionic centers can attack the electrophilic centers (carbonyl carbon or $\alpha$-carbon) intra-molecularly resulting in three possible products (XVI-XVIII, respectively). Product XVI, though is formed in a single step, seems less probable because of generation of strained fused heterocyclic system bearing - $\mathrm{COOH}$ group at bridge carbon of the fused system. The other two products, XVII and XVIII, seem more stable than XVI as well as XII. Hence, the exact structure to the degradation product detected in the study can be assigned through mass fragmentation/spectral data.

Ezetimibe is found to degrade through rearrangement of $\beta$-lactam ring to tetrahydropyrane ring in basic medium (Figure 17). ${ }^{142}$ There are two shortcomings in proposition of the structure through this rearrangement. Firstly, an aliphatic C-N bond is cleaved while a $\beta$-lactam amide bond remains intact, which is much more susceptible to hydrolysis than the $\mathrm{C}-\mathrm{N}$ bond. Secondly, no mass fragmentation or spectral data is given in support of formation of this product. So, herein, we propose that under hydrolytic conditions, the $\beta$-lactam ring opens due to hydrolysis of the amide bond (which is highly susceptible in alkaline medium as per the stan- dard literature) to form a carboxylic acid intermediate (XIX). It exits as carboxylate in alkaline medium, and its nucelophilic oxygen displaces the -OH group to form a lactonic product $(\mathrm{XX})$, which is stable than the $\beta$-lactam drug itself in alkaline medium.

Diclofenac, flupirtine and quinapril degrade to cyclized products, and formation of these products are reported to occur through formation of an amide bond between a carboxylic acid and an amino group in acidic medium (Figure 18). ${ }^{141,51,71}$ However, these routes seem improbable because the amino group is protonated in acidic medium destroying the nucleophilic character of amino nitrogen. Moreover, in diclofenac, presence of two chloro groups ortho to the -NH- group further intensifies the electron deficiency on the nitrogen. Hence, there is a need to study the products of these drugs to propose their unambiguous structures.

Indinavir degrades in acid hydrolytic condition through hydrolysis of an amide bond followed by condensation between the $-\mathrm{OH}$ and the free $-\mathrm{COOH}$ groups (Figure 19). ${ }^{53}$ This product is possible only if hydrolysis of amide bond 2 occurs in prefence over amide bond 1 . The valid reasons to support this reported route are, (i) the amide bond 2 is sterically hindered in comparison to amide bond 1, and (ii) the i-butyl group in amide bond 1 makes the amide nitrogen less electron deficient that in turn decreases the electrophilicity of carbonyl carbon in the bond.

Tamsulosin is reported to degrade by a mechanism that involves abstraction of acidic proton from sulphonamide by $-\mathrm{OH}$ resulting in formation of anion that attacks ether containing phenyl carbon resulting in the release of methanol to form a four membered ring (Figure 20). ${ }^{138}$

Reactions at active methylene group: A methylene group is said to be "active", when it is flanked by two strong electron withdrawing groups and/or elements. The carbon atom of such a methylene group is electron deficient, and the hydrogens attached to it become acidic. Ropinirole under alkaline condition form a hydrolyzed product due to the reactivity of active methylene group in the drug molecule through the mechanism as given in Figure 21. ${ }^{75}$ The product XXI is formed by the attack of $\mathrm{OH}^{-}$at active methylene carbon resulting in cleavage of $\gamma$-lactam ring followed by loss of carbon monoxide. Ornidazole is converted into an oxirane product under alkaline hydrolytic condition due to the reactivity of active methylene group. ${ }^{120}$ The most plausible mechanism for this reaction is outlined in Figure 22.

Dehydrogenation: It is one of the most unusual reactions that can occur in a hydrolytic medium. Benzopyridooxathiazepine is reported to degrade under acidic 
hydrolytic condition to a dehydrogenated product (XXII) by a mechanism that involves $\mathrm{N}$-protonation followed by rapid dehydrogenation that leads to the formation of corresponding iminium that further reacts by Michael addition of $\mathrm{H}_{2} \mathrm{O}$ followed by dehydration. ${ }^{26}$ However, formation of imimiun by this route seems improbable because of the following reasons: In the first step, the pyridine nitrogen is quaternized by protonation, which is accepted to occur in the acidic medium. But subsequently, $\mathrm{H}_{2} \mathrm{O}$ molecule is shown to abstract the $\alpha$-proton triggering electronic movements leading to loss of proton from the quaternized nitrogen, which is accepted by $\mathrm{Cl}$. Now, here water is a weak base and the $\alpha$-methylene is very weak acid. The spontaneity of reaction between such a weak acid and weak base is very less. Secondly, $\mathrm{Cl}^{-}$is a weak conjugate base of $\mathrm{HCl}$ (strong acid) and therefore its propensity to accept a proton to form $\mathrm{HCl}$ again is very remote. Thus, an alternate route of degradation of benzopyridooxathiazepine is proposed (Figure 23), on the basis of explanation by Baertschi and Alsante ${ }^{14}$ for a similar degradation, that involves oxidation of tertiary nitrogen to N-oxide (oxidation of tertiary amines is well reported and already discussed in preceding section), followed by protonation in acidic medium to form hydroxylamine. The latter undergoes dehydration to form iminium, which subsequently loses proton to form XXII.

Darifenacin is reported to degrade to a dehydrogenated product XXIII by dehydrogenation in furan ring (Figure 23) ${ }^{35}$ Though tetrahydrofuran or dihydrofuran does undergo air oxidation to furan but in the present case, the dehydrogenation from the dihyrofuran ring seems less probable because of presence of tertiary nitrogen in the form of pyrollidine. Nitrogen being a better nucleophile than oxygen, tends to undergo protonation as well as oxidation preferentially. Thus, the degradation of darifenacin can be proposed to occur by dehydrogenation that involves nitrogen atom by a similar mechanism as proposed for benzopyridooxathiazepine to form a dehydrogenated product XXIV (Figure 23). Both XXIII and XXIV have the same accurate molecular mass, and the actual structure can be ascertained through mass fragmentation data.

Reduction of carboxylic acid: This reaction is exactly opposite to dehydrogenation, which is discussed just in the preceeding section. A carboxylic acid is known to reduce to a primary alcohol in the presence of reducing agents. However, zofenopril is reported to reduce under alkaline hydrolytic condition (Figure 24). ${ }^{85}$ For this, neither any mechanism is proposed in the report nor there any literature support. Hence, there is a need to analyze, and probably revise, the proposed route for formation of this product from zofenopril.

Decarboxylation: An electron withdrawing group at $\alpha$ - $/ \beta$-position or $\alpha, \beta$-conjugated double bond in some aliphatic carboxylic acids makes them susceptible to decarboxylation either by $\mathrm{S}_{\mathrm{E}} 1$ or $\mathrm{S}_{\mathrm{E}} 2$ mechanism. ${ }^{105}$ Ambrisentan is reported to undergo decarboxylation in acidic hydrolytic medium ${ }^{89}$ by the mechanism as outlined in Figure 25. Protonation of the carboxylic acid of ambrisentan in acidic medium makes the carbonyl carbon a strong electrophile. It attracts nucleophilic attack of oxygen of the adjacent methoxy group resulting in formation of the product with the release of methanol and carbon dioxide. Ketorolac is reported to degrade by a mechanism (Figure 25), that involves the abstraction of proton from water molecule by carbonyl oxygen of carboxylic acid in acidic medium followed by dehydration and removal of formic acid ${ }^{145}$ However, from a chemist's viewpoint, this reaction is not feasible as the probability of abstraction of proton from acid is more than from water molecule. Thus, an alternate mechanism may be involved that involves the protonation of carbonyl oxygen in acidic medium followed by release of a proton and formic acid (Figure 25).

Ring opening: Heterocyclic rings in many drugs are opened under hydrolytic conditions resulting in degradation of drugs. Isoxazole undergoes dehydration under alkaline condition to generate a cyano-enol product that tautomerizes to a more stable cyano-keto product. ${ }^{146}$ This type of reaction has been reported in degradation of leflunomide (Figure 26). ${ }^{147}$ In ampicillin (148) and flucloxacillin, ${ }^{149}$ the bicyclic ring ( $\beta$-lactam ring fused with thiazolidine ring) is highly unstable and cleaves to form an acyclic structure under alkaline condition. The mechanism involves the attack of $\mathrm{OH}^{-}$ions at carbonyl carbon of $\beta$-lactam ring to form a germinal diol intermediate that collapses by losing $\mathrm{CO}_{2}$ to yield an intermediate (XXV). The latter is attacked by $\mathrm{OH}^{-}$ions at electron deficient carbon (carbon flanked by $-\mathrm{NH}-$ and -S-groups) resulting in the cleavage of C-S bond of ring B (Figure 27).

\section{CONCLUSIONS}

Degradation of a drug under hydrolytic conditions is largely influenced by the nature of functional groups across or around the susceptible bonds or functional groups in the drug molecule. In addition to the conventional reactions such as hydrolysis of amide, ester, ether, urethane and anhydride linkages, and of nitrile group, some unusual reactions also occur in a drug molecule under hydrolytic conditions. Such unusual/unconventional 
hydrolytic reactions are not reported well in literature. Only some of the reports on hydrolytic degradation studies on drugs have attempted to outline mechanisms for some of such unconventional hydrolytic reactions. A critical analysis of available reports has revealed that some degradation products are characterized and proposed to form through a route which does not seem probable. With a chemist's viewpoint that a product cannot be more unstable than the reactant under a given reaction conditions, the most probable mechanisms/routes for formations of unconventional hydrolytic products are proposed and discussed as well as alternate but more probable and justifiable structures of some seemingly unjustified unconventional hydrolytic products are proposed. These data are compiled and discussed to assist an analyst to predict all possible (conventional/unconventional) degradation products/impurities that may form from drug in hydrolytic conditions during the forced degradation studies.

\section{ACKNOWLEDGEMENT}

The authors are thankful to Punjabi University, Patiala for providing a wonderful library and free access to all kind of scientific literature, which makes it possible to compile this article.

\section{CONFLICT OF INTEREST}

There is no conflict of interest.

\section{ABBREVIATION USED}

WHO: World Health Organization; US-FDA: United States Food and Drug Administration; EMEA: European Medicines Agency; ICH: International Conference on Harmonisation; TPD: Therapeutic Products Directorate; TGA: Therapeutic Goods Administration; PRIs: Process related impurities; DRIs: Degradation related impurities; ACN: Acetonitrile; CAI: Carbamic acid intermediate; PAA: Phenyl acrylic acid; RT: Room temperature.

\section{REFERENCES}

1. EMEA/HPMC/CHMP/CVMP, Quality of Combination Herbal Medicinal Products/Traditional Herbal Medicinal Products. In: Committee on Herbal Medicinal Products, EMEA, London (UK); 2008.

2. EMEA/CHMP/QWP, Guideline on the Limits of Genotoxic Impurities. In: Committee for Medicinal Products for Human Use, EMEA, London (UK); 2006.

3. ICH, Impurities in new drug substances Q3A (R2). In: International Conference on Harmonisation, IFPMA, Geneva (Switzerland); 2006.

4. $\mathrm{ICH}$, Impurities in new drug products $\mathrm{Q} 3 \mathrm{~B}(\mathrm{R} 2)$. In: International Conference on Harmonisation, IFPMA, Geneva (Switzerland); 2006.

5. TPD, Impurities in Existing Drug Substances and Products. In: Therapeutic Products Directorate, Health Canada, Canada; 2005
6. TGA, Impurities in drug substances and drug products (Guidance 18). In: Therapeutic Goods Administration, DHA, Australia; 2013.

7. $\mathrm{ICH}$, Impurities Guideline for residual solvents Q3C (R5). In: International Conference on Harmonisation, IFPMA, Geneva (Switzerland); 2011.

8. $\mathrm{ICH}$, Impurities Guideline for metal impurities Q3D. In: International Conference on Harmonisation, IFPMA, Geneva (Switzerland); 2009.

9. $\mathrm{ICH}$, Stability testing of new drug substances and products Q1A (R2). In: International Conference on Harmonisation, IFPMA, Geneva (Switzerland); 2003.

10. Mabey $\mathrm{W}$ and Mill T. Critical review of hydrolysis of organic compounds in water under environmental conditions. J Phys Chem Ref Data.1978;7(2):383-415. http://dx.doi.org/10.1063/1.555572.

11. Sarmah AK and Sabadie J. Hydrolysis of sulfonylurea herbicides in soils and aqueous solutions: a review. J Agric Food Chem. 2002;50(22):6253-6265. http://dx.doi.org/10.1021/jf025575p ; PMid:12381100.

12. [12] Wyman CE, Decker SR, Himmel ME, Brady JW, Skopec CE, et al. Hydrolysis of cellulose and hemicelluloses. In: Dumitriu S, ed. Polysaccharides: Structural diversity and functional versatility. Boca Raton: Taylor \& Francis Group; 2004.995-1034. http://dx.doi.org/10.1201/9781420030822.ch43.

13. Engineer C, Parikh J and Raval A. Review on hydrolytic degradation behavior of biodegradable polymers from controlled drug delivery system. Trends Biomater Artif Organs 2011;25(2):79-85.

14. Baertschi SW and Alsante KM. Stress Testing: The Chemistry of Drug Degradation. In: Baertschi SW, ed. Pharmaceutical Stress Testing: Predicting Drug Degradation. Boca Raton: Taylor \& Francis Group; 2005.51-72. http://dx.doi.org/10.1201/9780849359194.ch3 http://dx.doi. org/10.1201/9780849359194

15. Saini $B$ and Bansal G. Degradation study on sulfasalazine and a validated HPLCUV method for its stability testing. Sci Pharm 2014;82(2):295-306. http://dx.doi. org/10.3797/scipharm.1311-15; PMid:24959403:PMCid:PMC4065124.

16. Bansal G, Singh M, Jindal KC and Singh S. Ultraviolet-photodiode array and high-performance liquid chromatographic/mass spectrometric studies on forced degradation behavior of glibenclamide and development of a validated stability-indicating method. JAOAC Int. 2008;91(4):709-719. PMid:18727528.

17. Bansal G, Singh $M$ and Jindal KC. Forced degradation study on gliclazide and application of validated stability-indicating HPLC-UV method in stability testing of gliclazide tablets. Chromatographia 2007;66(9-10):751-755. http:// dx.doi.org/10.1365/s10337-007-0394-4.

18. Bansal G, Singh M, Jindal KC and Singh S. LC-UV-PDA and LC-MS studies to characterize degradation products of glimepiride. J Pharm Biomed Anal 2008;48(3):788-795. http://dx.doi.org/10.1016/j.jpba.2008.08.003; PMid:18804934.

19. Bansal G, Singh M, Jindal KC and Singh S. LC and LC-MS study on establishment of degradation pathway of glipizide under forced degradation conditions. J Chromatogr Sci 2008;46(6):510-517. http://dx.doi.org/10.1093/ chromsci/46.6.510; PMid:18647472.

20. Srinivasu P, SubbaRao DV, Vegesna RVK and Babu KS. A validated stability-indicating LC method for acetazolamide in the presence of degradation products and its process-related impurities. J Pharm Biomed Anal. 2010;52(1):142-148. http://dx.doi.org/10.1016/j.jpba.2009.12.011; PMid:20053517.

21. Tamaro I, Aprile S, Giovenzana GB and Grosa G. Development and validation of a stability-indicating HPLC-UV method for the determination of alizapride and its degradation products. J Pharm Biomed Anal. 2010;51:1024-1031. http://dx.doi.org/10.1016/j.jpba.2009.10.026 ; PMid:19962264.

22. Stoiljkovic ZZ, Jadranin MB, Duric SLJ, Petrovic SD, Avramovivic ML, et al. Investigation of forced and total degradation products of amlodipine besylate by liquid chromatography and liquid chromatography-mass spectrometry. Chem Ind Chem Eng Q. 2014; 20(2):295-304. http://dx.doi.org/10.2298/ CICEQ121226011S.

23. Tiwari RN, Shah N, Bhalani V and Mahajan A. LC, MSn and LCMS/MS studies for the characterization of degradation products of amlodipine. J Pharm Anal. 2015;5(1):33-42. http://dx.doi.org/10.1016/j. jpha.2014.07.005.

24. Subba Rao DV, Radhakrishnanand $P$, Surendranath $K V$, Raghuram $P$ and Himabindu V. A stability indicating LC method for amtolmetin guacyl. Chromatographia. 2008;68(7-8):567-577. http://dx.doi.org/10.1365/s10337008-0743-y. 
25. Narayanam M, Sahu A and Singh S. Characterization of stress degradation products of benazepril by using sophisticated hyphenated techniques. J Chromatogr A. 2014;1271(1):124-136. http://dx.doi.org/10.1016/j.chroma. 2012.11.044; PMid:23237710.

26. Lecoeur M, Verones V, Vaccher C, Bonte JP, Lebegue N, et al. Structural elucidation of degradation products of a benzopyridooxathiazepine under stress conditions using electrospray orbitrap mass spectrometry - Study of degradation kinetic. Eur J Pharm Sci. 2012;45(5):559-569. http://dx.doi. org/10.1016/j.ejps.2011.12.009; PMid:22207039.

27. Rao RN, Raju $A N$ and Narsimha R. Isolation and characterization of process related impurities and degradation products of bicalutamide and development of RP-HPLC method for impurity profile study. J Pharm Biomed Anal. 2007;46:505-519.

28. Bansal G, Singh R, Saini B and Bansal Y. ESI-MSn and LC-ESI-MS studies to characterize forced degradation products of bosentan and a validated stability-indicating LC-UV method. J Pharm Biomed Anal. 2013;72:186-197. http://dx.doi.org/10.1016/j.jpba.2012.08.014; PMid:22999302.

29. Wu C, Xu X, Feng C, Shi Y, Liu W, Zhu X et al. Degradation kinetics study of cabozantinib by a novel stability-indicating LC method and identification of its major degradation products by LC/TOF-MS and LC-MS/MS. J Pharm Biomed Anal. 2014;98:356-363. http://dx.doi.org/10.1016/j.jpba.2014.06.008; PMid:24992215.

30. Rao DVS, Radhakrishnanand P, Suryanarayana MV and Himabindu V. A stability indicating Ic method for candesartan cilexetil. Chromatographia. 2007;66(7-8):499-507. http://dx.doi.org/10.1365/s10337-007-0364-x.

31. Rao RN, Ramakrishna K, Sravan B and Santhakumar K. RP-HPLC separation and ESI-MS, $1 \mathrm{H}-$, and 13C-NMR characterization of forced degradants including process related impurities of carisbamate: Method development and validation. J Pharm Biomed Anal. 2013;77:49-54. http:// dx.doi.org/10.1016/j.jpba.2013.01.001; PMid:23376724.

32. Ebeid WM, Elkady EF, El-Zaher AA, El-Bagary RI and Patonay G. Stabilityindicating RP-LC method for determination of azilsartan medoxomil and chlorthalidone in pharmaceutical dosage forms: application to degradation kinetics. Anal Bioanal Chem. 2014;406(26):6701-6712. http://dx.doi. org/10.1007/s00216-014-8085-0 ; PMid:25190009.

33. Elkady EF and Fouad M.A. Forced degradation study to develop and validate stability indicating RP-LC method for the determination of ciclesonide in bulk drug and metered dose inhalers Talanta. 2011;87:222-229. http://dx.doi. org/10.1016/j.talanta.2011.10.001; PMid:22099671.

34. Mashelkar UC and Renapurkar SD. A LC-MS compatible stability-indicating HPLC assay method for clopidogrel bisulphate. Int J Chem Tech Res. 2010;2(2):822-829.

35. Murthy MV, Krishnaiah C, Srinivas K, Rao KS and Kumar NR. Development and validation of RP-UPLC method for the determination of darifenacin hydrobromide, its related compounds and its degradation products using design of experiments. J Pharm Biomed Anal. 2013;72:40-50. http://dx.doi. org/10.1016/j.jpba.2012.09.013; PMid:23146225.

36. Rao RN, Ramachandra B, Sravan B an Khalid S. LC-MS/MS structural characterization of stress degradation products including the development of a stability indicating assay of Darunavir: An anti-HIV drug. J Pharm Biomed Anal. 2014;89:28-33. http://dx.doi.org/10.1016/j.jpba.2013.10.007; PMid:24252722.

37. Hadad GM. Validated stability-indicating HPLC method for the determination of dimethyl-4,4'-dimethoxy-5,5,5',6'-dimethylene dioxybiphenyl-2,2'-dicarboxylate (DDB) and its degradation products. J Pharm Biomed Anal. 2008;47(4):695703.http://dx.doi.org/10.1016/j.jpba.2008.02.017; PMid:18403161,

38. Kumar D, Tomar RS, Deolia SK, Mitra M, Mukherjee R, et al. Isolation and characterization of degradation impurities in docetaxel drug substance and its formulation. J Pharm Biomed Anal. 2007;43(4):1228-1235. http://dx.doi. org/10.1016/j.jpba.2006.10.015 ; PMid:17126518.

39. Rao RN, Naidu CG, Prasad KG, Santhakumar B and Saida S. Development and validation of a stability indicating assay of doxofylline by RP-HPLC: ESIMS/MS, $1 \mathrm{H}$ and 13C NMR spectroscopic characterization of degradation products and process related impurities. J Pharm Biomed Anal. 2013; 78-79:92-99. http://dx.doi.org/10.1016/j.jpba.2013.02.004 ; PMid:23466440.

40. Chaudhari R, Mohanraj K and Shirsat V. MS/MS and HPLC characterization of forced degradation products of dutasteride and tamsulosin hydrochloride. Int J Pharm Sci Res. 2014; 5(7):2791-2806.
41. Montgomery ER, Edmanson AL, Cook SC and Hovsepian PK. Development and validation of a reverse-phase HPLC method for analysis of efavirenz and its related substances in the drug substance and in a capsule formulation. J Pharm Biomed Anal. 2001;25(2):267-284. http://dx.doi.org/10.1016/S07317085(00)00495-7.

42. Toporisic R, Mlakar A, Hvala J, Prislan I and Kralj LZ. Identification of new impurities of enalapril maleate on oxidation in the presence of magnesium monoperoxyphthalate. J Pharm Biomed Anal. 2010;52(2):294-299. http:// dx.doi.org/10.1016/j.jpba.2009.12.018; PMid:20122808.

43. Bhardwaj SP and Singh S. Study of forced degradation behavior of enalapril maleate by LC and LC-MS and development of a validated stabilityindicating assay method. J Pharm Biomed Anal. 2008;46(1):113-120. http:// dx.doi.org/10.1016/j.jpba.2007.09.014; PMid:17964751.

44. Sonawane $S$ and Gide P. An experimental design approach for the forced degradation studies and development of a stability-indicating LC method for eplerenone in tablets. J Liq Chromatogr Relat Technol. 2011;34(17):20202031. http://dx.doi.org/10.1080/10826076.2011.582913.

45. Srinivas M, Avupati NR, Sait S and Mukkanti K. Stability indicating HPLC method for the determination of eslicarbazepine acetate and its impurities in bulk drugs and pharmaceutical dosage forms. J Liq Chromatogr Relat Technol. 2012;35(11):1550-1564.

46. Peraman R, Nayakanti D, Dugga HHT and Kodikonda S. Development and validation of a stability-indicating assay of etofenamate by rp-hplc and characterization of degradation products. Sci Pharm. 2013;81(4):1017-1028. http://dx.doi.org/10.3797/scipharm.1305-19PMid:24482770:PMCid:PMC3867237.

47. Raman NVVSS, Harikrishna KA, Prasad AVVS, Reddy KR and Ramakrishna K. Development and validation of a stability-indicating RP-LC method for famciclovir. J Pharm Biomed Anal. 2009;50(5):797-802. http://dx.doi. org/10.1016/j.jpba.2009.06.031; PMid:19632799.

48. Salama FMM, Nassar MWI, El-Din MMKS, Attia KAM and Kaddah MY. Determination of fenofibrate and the degradation product using simultaneous UV-derivative spectrometric method and HPLC. Am J Anal Chem. 2011;2(03):332-343. http://dx.doi.org/10.4236/ajac.2011.23041.

49. Sangoi MS, Todeschini $V$ and Steppe M. Fesoterodine stress degradation behavior by liquid chromatography coupled to ultraviolet detection and electrospray ionization mass spectrometry Talanta. 2011;84(4):1068-1079. http://dx.doi.org/10.1016/j.talanta.2011.03.018; PMid:21530781.

50. Mistiri F, Louati K, Grissa O, Kallel M and Safta F. Study of forced degradation behaviour of florfenicol by LC and LC-MS and development of a validated stability-indicating assay method. Ann Pharm Fr. 2012;70(6):333-347. http:// dx.doi.org/10.1016/j.pharma.2012.08.004 ; PMid:23177560.

51. Peraman R, Lalitha KV, Raja B. NM and Routhu HB. Identification of degradation products and a stability-indicating RP-HPLC method for the determination of flupirtine maleate in pharmaceutical dosage forms. Sci Pharm. 2014;82(2):281-293. http://dx.doi.org/10.3797/scipharm.1310-01; PMid:24959399:PMCid:PMC4065123.

52. Narayanam $\mathrm{N}$ and Singh $\mathrm{S}$. Characterization of stress degradation products of fosinopril by using LC-MS/TOF, MSn and on-line H/D exchange. J Pharm Biomed Anal. 2014;92:135-143. http://dx.doi.org/10.1016/j.jpba.2014.01.010; PMid:24518132.

53. Rao RN, Vali RM and Raju SS. Liquid chromatography tandem mass spectrometric studies of indinavir sulphate and its forced degradation products. J Pharm Biomed Anal. 2013;74:101-110. http://dx.doi.org/10.1016/j. jpba.2012.10.025 ; PMid:23245240.

54. Kasawar GB and Farooqui M. Development and validation of a stability indicating RP-HPLC method for the simultaneous determination of related substances of albuterol sulfate and ipratropium bromide in nasal solution. J Pharm Biomed Anal. 2010;52(1):19-29. http://dx.doi.org/10.1016/j. jpba.2009.11.026; PMid:20045275.

55. Patel PN, Borkar RM, Kalariya PD, Gangwal RP, Sangamwar AT, et al. Characterization of degradation products of ivabradine by LC-HR-MS/MS: a typical case of exhibition of different degradation behaviour in $\mathrm{HCl}$ and H2SO4 acid hydrolysis. J Mass Spectrom. 2015;50(2):344-353. http://dx.doi. org/10.1002/jms.3533; PMid:25800016.

56. Belal F, Elbrashy A, Eid M and Nasr JJ. Stability indicating LC method for the determination of lacidipine in tablets. Application to degradation kinetics and content uniformity testing. Chromatographia. 2009;69(11-12):1201-1209. http://dx.doi.org/10.1365/s10337-009-1067-2. 
57. Ramisetti NR, Kuntamukkala R, Lakshetti S and Sripadi P. Identification and characterization of stress degradants of lacosamide by LC-MS and ESI-QTOF-MS/MS: Development and validation of a stability indicating RP-HPLC method. J Pharm Biomed Anal. 2014;95:256-264. http://dx.doi.org/10.1016/j. jpba.2014.03.010; PMid:24699370.

58. Che X, Shen L, Xu H and Liu K. Isolation and characterization of processrelated impurities and degradation products in larotaxel. J Pharm Biomed Anal. 2011;55:1190-1196. http://dx.doi.org/10.1016/j.jpba.2011.03.036 ; PMid:21530132.

59. Seshachalam U, Haribabu B and Chandrasekhar KB. A novel validated LC method for quantitation of lopinavir in bulk drug and pharmaceutical formulation in the presence of its potential impurities and degradation products. Biomed Chromatogr. 2007;21(7):716-723. http://dx.doi.org/10.1002/bmc.810; PMid:17370253.

60. Walash MI, Belal F, El-Enany N, Eid M and El-Shaheny R.N. Stability-indicating micelle-enhanced spectrofluorimetric method for determination of loratadine and desloratadine in dosage forms. Luminescence. 2011;26(6):670-679. http://dx.doi.org/10.1002/bio.1294; PMid:21491578.

61. Modhave DT, Handa T, Shah RP and Singh S. Stress degradation studies on lornoxicam using LC, LC-MS/TOF and LC-MSn. J Pharm Biomed Anal. 2011;56(3):538-545. http://dx.doi.org/10.1016/j.jpba.2011.06.012; PMid:21757313.

62. Talluri MVNK, Dharavath S, Kalariya PD, Prasanth B and Srinivas R. Structural characterization of alkaline and oxidative stressed degradation products of lurasidone using LC/ESI/QTOF/MS/MS. J Pharm Biomed Anal. 2015;105:1-9. http://dx.doi.org/10.1016/j.jpba.2014.11.035 ; PMid:25527975.

63. Thomas S, Bharti A, Tharpa K and Agarwal A. Quantification of potential impurities by a stability indicating UV-HPLC method in niacinamide active pharmaceutical ingredient. J Pharm Biomed Anal. 2012;60:86-90. http:// dx.doi.org/10.1016/j.jpba.2011.10.033; PMid:22115882.

64. Sharma RN and Pancholi SS. RP-HPLC-DAD method for determination of olmesartan medoxomil in bulk and tablets exposed to forced conditions. Acta Pharm 2010;60(1):13-24. http://dx.doi.org/10.2478/v10007-010-0010-2; PMid:20228038.

65. Pathare DB, Jadhav AS and Shingare MS. A validated stability indicating LC method for oxcarbazepine. J Pharm Biomed Anal. 2007;43(5):1825-1830. http://dx.doi.org/10.1016/j.jpba.2006.12.002 ; PMid:17223301.

66. Canavesi R, Aprile S, Varese E and Grosa G. Development and validation of a stability-indicating LC-UV method for the determination of pantethine and its degradation product based on a forced degradation study. J Pharm Biomed Anal. 2014;97:141-150. http://dx.doi.org/10.1016/j.jpba.2014.04.025; PMid:24863372.

67. Bhimavarapu R, Chitra KP, Meda H, Kanikanti D, Anne M, et al. Forced degradation study of paracetamol in tablet formulation using RP-HPLC. Bull Pharm Res. 2011;1(3):13-17.

68. Mone MK and Chandrasekhar KB. Degradation Studies of pentoxifylline: Isolation and characterization of a novel gem-dihydroperoxide derivative as major oxidative degradation product. J Pharm Biomed Anal. 2010;53(3):335342. http://dx.doi.org/10.1016/j.jpba.2010.04.006 ; PMid:20466503.

69. Gawad DA and Belal TS. HPLC-DAD stability indicating determination of pentoxyverine citrate. Application to degradation kinetics and assay of syrup dosage form. Arab J Chem 2013 (in-press) http://dx.doi.org/10.1016/j. arabjc.2013.11.023. http://dx.doi.org/10.1016/j.arabjc.2013.11.023.

70. Hadad GM. Validated, Stability-Indicating LC-Method for analysis of pipenzolate bromide and its hydrolysis products. Chromatographia. 2008;68(3-4):207-212. http://dx.doi.org/10.1365/s10337-008-0715-2.

71. Dendeni M, Cimetiere N, Huguet S, Amrane A and Hamida NB. Forced degradation study of quinapril by UPLC-DAD and UPLC/MS/MS: Identification of by-products and development of degradation kinetics. Curr Pharm Anal. 2013;9(3):278-290. http://dx.doi.org/10.2174/1573412911309030006.

72. Rao RN, Ramachandra B, Vali RM and Raju SS. LC-MS/MS studies of ritonavir and its forced degradation products. J Pharm Biomed Anal. 2010;53(4):833-842. http://dx.doi.org/10.1016/j.jpba.2010.06.004 ; PMid:20619992.

73. Abdallah MA, Al-Ghobashy MA and Lotfy HM. Investigation of the profile and kinetics of degradation of Rivaroxaban using HPLC, TLC-densitometry and LC/MS/MS: Application to pre-formulation studies. Bull Fac Med Cario Univ. 2015;53(1):55-61. http://dx.doi.org/10.1016/j.bfopcu.2015.01.002.
74. Rao BM, Srinivasu MK, Kumar KP, Bhradwaj N, Ravi R, et al. A stability indicating LC method for rivastigmine hydrogen tartarate. J Pharm Biomed Anal. 2005;37(1):57-63. http://dx.doi.org/10.1016/j.jpba.2004.09.041; PMid:15664743.

75. Parmar G, Sharma S, Singh K and Bansal G. Forced degradation study to develop and validate stability-indicating RP-LC for quantification of ropinirole hydrochloride in its modified release tablets. Chromatographia. 2009;69:199-206. http://dx.doi.org/10.1365/s10337-008-0866-1.

76. Louati K, Mistiri F, Kallel M and Safta F. Stress degradation study on sulfadimethoxine and development of a validated stability-indicating HPLC assay. Ann Pharm Fr 2011;69(2):91-99. http://dx.doi.org/10.1016/j. pharma.2010.11.003 ; PMid:21440101.

77. Pathare DB, Jadhav AS and Shingare MS. A validated stability indicating RPLC method for tazarotene. Chromatographia. 2007;66:247-250. http:// dx.doi.org/10.1365/s10337-007-0265-z.

78. Erika DG, Silvio A and Giorgio G. Forced degradation study of thiocolchicoside: characterization of its degradation products. J Pharm Biomed Anal. 2012;61:215-223. http://dx.doi.org/10.1016/j.jpba.2011.12.008; PMid:22226415.

79. Jovic Z, Zivanovic L, Protic A, Radisic M, Lausevic M, et al. Forced degradation study of torasemide: characterization of its degradation products. J Liq Chromatogr Relat Technol. 2013;36(15):2082-2094.

80. Mehta S, Shah RP and Singh S. Strategy for identification and characterization of small quantities of drug degradation products using LC and LC-MS: Application to valsartan, a model drug. Drug Test Analysis. 2010;2(2):82-90. http://dx.doi.org/10.1002/dta.116.

81. Krishnaiah C, Reddy AR, Kumar R and Mukkanti K. Stability-indicating UPLC method for determination of valsartan and their degradation products in active pharmaceutical ingredient and pharmaceutical dosage forms. J Pharm Biomed Anal. 2010;53(3):483-489. http://dx.doi.org/10.1016/j. jpba.2010.05.022 ; PMid:20646890.

82. Kalariya PD, Talluri MVNK, Patel PN and Srinivas R. Identification of hydrolytic and isomeric N-oxide degradants of vilazodone by on line LCESI-MS/MS and APCI-MS. J Pharm Biomed Anal. 2015;102:353-365. http:// dx.doi.org/10.1016/j.jpba.2014.09.033 ; PMid:25459935.

83. Roskar R, Simoncic Z, Gartner A and Kmetec V. Stability of new potential ACE inhibitor in the aqueous solutions of different $\mathrm{pH}$. J Pharm Biomed Anal. 2009;49(2):295-303. http://dx.doi.org/10.1016/j.jpba.2008.11.029; PMid:19135817.

84. Zhou C, Liu WY, Feng F, Ye H and Wu CY. Determination and stress studies on YK-1101, a potential histone deacetylase, by HPLC-UV and HPLC-TOF/ MS methods. J Pharm Anal 2013;3(3):168-172. http://dx.doi.org/10.1016/j. jpha.2013.01.003.

85. Ramesh T, Rao PN and Rao RN. LC-MS/MS characterization of forced degradation products of zofenopril. J Pharm Biomed Anal. 2014;88:609-616. http://dx.doi.org/10.1016/j.jpba.2013.10.018; PMid:24211724.

86. Rao BM, Srinivasu MK, Sridhar G, Kumar PR, Chandrasekhar KB, et al. A stability indicating LC method for zolmitriptan. J pharm Biomed Anal. 2005;39:503-509. http://dx.doi.org/10.1016/j.jpba.2005.04.018 : http://dx.doi. org/10.1016/j.jpba.2005.04.012

87. Pushpalatha P, Sarin RK, Idris M, Rao MA, Baggi TRR, et al. Base hydrolytic forced degradation study of zolpidem tartrate by HPLC. J Chem Metrol. 2012;6(1):1-8.

88. Morrison RT, Boyd RN. Organic Chemistry. 6th ed. New Delhi: Prentice-Hall of India Private Limited; 1996.

89. Ramisetti NR, Kuntamukkala R. LC-MS/MS characterization of forced degradation products of ambrisentan: development and validation of a stability-indicating RP-HPLC method. New J Chem. 2014;38(7):3050-3061. http://dx.doi.org/10.1039/c4nj00075g.

90. Reddy PRM, Sreeramulu J, Naidu PY and Reddy AR. Stability indicating fast LC for the simultaneous estimation of intermediates and degradants of duloxetine hydrochloride. Chromatographia. 2010;71(1-2):95-100. http:// dx.doi.org/10.1365/s10337-009-1397-0.

91. Djabrouhou $\mathrm{N}$ and Guermouche $\mathrm{MH}$. Development of a stability-indicating HPLC method of etifoxine with characterization of degradation products by LC-MS/TOF, 1H and 13C NMR. J Pharm Biomed Anal. 2014;100:11-20. http://dx.doi.org/10.1016/j.jpba.2014.07.017 ; PMid:25117950.

92. KaushikD and Bansal G. Characterization of degradation products of idarubicin through LC-UV, MSn and LC-MS-TOF studies. J Pharm Biomed Anal. 2013;85:123-131. http://dx.doi.org/10.1016/j.jpba.2013.07.002; PMid:23933565. 
93. Dutta AK, Avery BA and Wyandt CM. Development and validation of a stabilityindicating reversed-phase high performance liquid chromatography method for NPC 1161C, a novel8-aminoquinolineanti-malarial drug. JChromatogrA. 2006; 1110(1):35-45. http://dx.doi.org/10.1016/j.chroma.2006.01.040; PMid:16483584.

94. Biro A, Pergel E, Arvai G, llisz I, Szepesi G, PeterAet al. High-performance liquid chromatographic study of topiramate and its impurities. Chromatographia. 2006;63(13):S137-S141 http://dx.doi.org/10.1365/s10337-006-0818-6.

95. Bansal G, Maddhesia PK and Bansal Y. MS2 /TOF and LC-MS /TOF studies on toremifene to characterize its forced degradation products Analyst. 2011;136(24):5218-5228. http://dx.doi.org/10.1039/c1an15587c; PMid:22013582.

96. Baertschi SW and Jansen PJ. Stress Testing: A Predictive Tool. In: Baertschi SW, ed. Pharmaceutical Stress Testing: Predicting Drug Degradation. Boca Raton: Taylor \& Francis Group. 2005.36-39. http://dx.doi. org/10.1201/9780849359194 .

97. Sitaram C, Rupakula R and Reddy B.N. Determination and characterization of degradation products of anastrazole by LC-MS/MS and NMR spectroscopy. J Pharm Biomed Anal. 2011;56:962-968. http://dx.doi.org/10.1016/j. jpba.2011.08.022 ; PMid:21890298.

98. Sharma M, Jawa PR, Gill RS and Bansal G. Citalopram Hydrobromide: Degradation Product Characterization and a Validated Stability-Indicating LC-UV Method. J Braz Chem Soc. 2011; 22(5):836-848. http://dx.doi. org/10.1590/S0103-50532011000500005.

99. Sridhar L, Goutami P, Darshan DV, Ramakrishna K, Rao RN, et al. LC-ESIMS/MS studies on saxagliptin and its forced degradation products. Anal Methods. 2014;6(20):8212-8221. http://dx.doi.org/10.1039/C4AY01152J.

100. Metwally FH, Abdelkawy $M$ and Abdelwahab NS. Application of spectrophotometric, densitometric, and HPLC techniques as stability indicating methods for determination of zaleplon in pharmaceutical preparations. Spectrochim. Acta. 2007;68(5):1220-1230. http://dx.doi. org/10.1016/j.saa.2007.01.024; PMid:17353143.

101. Zhou YX, Zhou WT, Sun LL, Zou QG, Wei P, et al. Characterization of process-related impurities including forced degradation products of alogliptin benzoate and the development of the corresponding reversed-phase highperformance liquid chromatography method. J Sep Sci. 2014;37(11):1248-1255. http://dx.doi.org/10.1002/jssc.201301384; PMid:24616424.

102. Seshaiah KR, Samanta SK, Reddy VK and Prasadaraju VVNKV. A stabilityindicating liquid chromatographic method for lomustine. J Pharm Biomed Anal. 2011;54(1):213-216. http://dx.doi.org/10.1016/j.jpba.2010.07.020; PMid:20719460.

103. Pawar SM, Khatal LD, Gabhe SY and Dhaneshwar SR. LC-UV and LC-MS evaluation of stress degradation behavior of desvenlafaxine. J Pharm Anal. 2012;2(4):264-271. http://dx.doi.org/10.1016/j.jpha.2012.02.009.

104. Bianchini RM, Castellano PM and Kaufman TS. Validated stability-indicating HPLC method for the determination of pridinol mesylate. Kinetics study of its degradation in acid medium. J Pharm Biomed Anal. 2008;48:1151-1160. http://dx.doi.org/10.1016/j.jpba.2008.09.005 ; PMid:18922659.

105. March J. Advanced Organic Chemistry: Reactions, Mechanisms and Structure, 4th ed. New Delhi: John Wiley \& Sons; 2009.

106. Schone L, Schindelka J, Szeremeta E, Schaefer T, Hoffmann D, Rudzinski KJ et al., Atmospheric aqueous phase radical chemistry of the isoprene oxidation products methacrolein, methyl vinyl ketone, methacrylic acid and acrylic acid - kinetics and product studies. Phys. Chem. Chem. Phys., 2014;16(13):62576272. http://dx.doi.org/10.1039/c3cp54859g ; PMid:24569503

107. Chen Q, Zielinski D, Chen J, Koski A, Werst D, et al. A validated, stabilityindicating HPLC method for the determination of dexamethasone-coated drug-eluting stents. J Pharm Biomed Anal. 2008;48(3):732-738. http://dx.doi. org/10.1016/j.jpba.2008.07.010 ; PMid:18722070

108. Seshachalam U, Haribabu B and Chandrasekhar KB. Development and validation of a stability-indicating liquid chromatographic method for determination of emtricitabine and related impurities in drug substance. J Sep Sci. 2007;30(7):999-1004. http://dx.doi.org/10.1002/jssc.200600429 ; PMid:17566333

109. Nalwade SU, Reddy VR, Rao DD and Morisetti NK. A validated stability indicating ultra performance liquid chromatographic method for determination of impurities in esomeprazole magnesium gastro resistant tablets. J Pharm Biomed Anal. 2012;57:109-114. http://dx.doi.org/10.1016/j.jpba.2011.08.025; PMid:21920688
110. Murthy MV, Srinivas K, Kumar R and Mukkanti K. Development and validation of a stability-indicating LC method for determining palonosetron hydrochloride, its related compounds and degradation products using naphthalethyl stationary phase. J Pharm Biomed Anal. 2011;56(2):429-435. http://dx.doi.org/10.1016/j.jpba.2011.05.037 ; PMid:21708445.

111. Lew A, Gao $Q$ and Takahashi L.H. Analysis and identification of prochlorperazine impurities and forced degradation products using a stabilityindicating HPLC method. J Liq Chromatogr Relat Technol. 2011; 34(8):634-651. http://dx.doi.org/10.1080/10826076.2011.558650.

112. Rotival R, Espeau P, Corvis $Y$, Guyon F and Do B. Determination of quinacrine dihydrochloride dihydrate stability and characterization of its degradants. J Pharm Sci. 2011;100(8):3223-3232. http://dx.doi.org/10.1002/jps.22543; PMid:21425260.

113. Buchireddy R, Mukkanti K, Srinivasulu $P$ and Srinivas KSV. A Validated, Stability-Indicating, LC method for Rabeprazole Sodium. Chromatographia. 2008;68(3-4):275-280. http://dx.doi.org/10.1365/s10337-008-0670-y.

114. Prakash L, Himaja M, Subbaiah BV, Vasudev R, Srinivasulu C, et al. Isolation, identification and characterization of degradant impurities in tolterodine tartrate formulation. J Pharm Biomed Anal. 2014;90:215-221. http://dx.doi. org/10.1016/j.jpba.2013.12.007 ; PMid:24384498.

115. Parker RE and Issacs NS. Mechanisms of epoxide reactions. Chem Rev. 1959;59(4):737-799. http://dx.doi.org/10.1021/cr50028a006.

116. Song Z, Chen X, Zhang D, Ren L, Fang L, Cheng W et al. Kinetic study of the degradation of PAC-1 and identification of a degradation product in alkaline condition. Chromatographia. 2009;70(11-12):1575-1580. http:// dx.doi.org/10.1365/s10337-009-1348-9.

117. El-Houssini OM. Study of forced degradation behavior of idrocilamide and development of stability indicating LC method. J Anal. Chem. 2014; 69(10):1003-1008. http://dx.doi.org/10.1134/S1061934814100050.

118. Golubovic JB, Protic AD, Zecevic ML and Otasevic BM. Quantitative structure retention relationship modeling in liquid chromatography method for separation of candesartan cilexetil and its degradation products. Chemometr Intell Lab Syst. 2015;140:92-101. http://dx.doi.org/10.1016/j. chemolab.2014.11.005.

119. AlH, Gosmann G, Schneider PH and Bergold AM. LC stability studies of voriconazole and structural elucidation of its major degradation product. Chromatographia. 2009;69(2):S115-S122.

120. Bakshi M, Singh $B$, Singh $A$ and Singh $S$. The $I C H$ guidance in practice: stress degradation studies on ornidazole and development of a validated stability-indicating assay. J Pharm Biomed Anal. 2001;26:891-897. http:// dx.doi.org/10.1016/S0731-7085(01)00475-7.

121. Kumar KK, Rao CK, Lakshmi MV and Mukkanti K. A validated stability indicating RP-UPLC method for atorvastatin calcium. Am J Anal. Chem. 2012;3:392-399. http://dx.doi.org/10.4236/ajac.2012.35052.

122. Sawant $S$ and Ghante M. A validated stability indicating RP-HPLC method for simvastatin, identification and characterization of forced degradation products of simvastatin using LC-ESI-MS/MS, Adv Analyt Chem. 2015;5(1):8-16.

123. Oliveira PR, Bernardi LS, Mendes C, Sangoi MS and Silva MAS. Liquid chromatographic determination of lumiracoxib in pharmaceutical formulations. J Pharm Biomed Anal. 2010;51:728-732. http://dx.doi. org/10.1016/j.jpba.2009.09.008; PMid:19800190

124. Petersen-Mahrt SK, Neuberger MS. In vitro deamination of cytosine to uracil in single-stranded DNA by apolipoprotein B editing complex catalytic subunit 1 (APOBEC1). J Biol Chem. 2003; 278(22):19583-19586. http://dx.doi. org/10.1074/jbc.C300114200; PMid:12697753.

125. Yao L, Cukier RI, Yan H. Catalytic mechanism of guanine deaminase: An ONIOM and molecular dynamics study. J Phys Chem. 2007; 111(16):4200-4210. http://dx.doi.org/10.1021/jp0673056 ; PMid:17394305.

126. Rao RN, Vali RM, Ramachandra B and Raju SS. Separation and characterization of forced degradation products of abacavir sulphate by LCMS/MS. J Pharm Biomed Anal. 2011;54:279-285. http://dx.doi.org/10.1016/j. jpba.2010.08.021; PMid:20869185.

127. Patil PM, Wankhede SB and Chaudhari PD. A validated stability indicating HPTLC method for estimation of acyclovir in tablets in presence of its alkaline hydrolysis degradation product. Bull Fac Med Cario Univ. 2014;52(2):245-257. http://dx.doi.org/10.1016/j.bfopcu.2014.09.002. 
128. Srinivas KSV, Buchireddy R, Madhusudhan G, Mukkanti K and Srinivasulu P. Stress degradation studies on aripiprazole and development of a validated stability indicating LC method. Chromatographia. 2008;68(7-8):635-640. http://dx.doi.org/10.1365/s10337-008-0739-7.

129. Subbaiah BV, Ganesh KKS, Krishna GV, Vyas K, Dev RV, et al. Isolation and characterisation of degradant impurities in Dipyridamole formulation. J Pharm Biomed Anal. 2012;61:256-264. http://dx.doi.org/10.1016/j.jpba.2011.11.028; PMid:22206889

130. Krishna MV, Dash RN, Reddy BJ, Venugopal P, Sandeep P, et al. Quality by design $(\mathrm{QbD})$ approach to develop HPLC method for eberconazole nitrate: Application to hydrolytic, thermal, oxidative and photolytic degradation kinetics. J Saudi Chem Soc (in-press). http://dx.doi.org/10.1016/j. jscs.2012.12.001; http://dx.doi.org/10.1016/j.jscs.2012.12.001

131. Chakravarthy VA, Sailaja BBV and Kumar AP. Stability-indicating RP-HPLC method for simultaneous estimation of enrofloxacin and its degradation products in tablet dosage forms. J Anal Methods Chem. 2015;1-11. http:// dx.doi.org/10.1155/2015/735145 ; PMid:25705547 PMCid:PMC4326284.

132. Ramesh T, Rao PN and Rao RN. LC-MS/MS method for the characterization of the forced degradation products of entecavir. J Sep Sci. 2014;37:368-375. http://dx.doi.org/10.1002/jssc.201300959; PMid:24323372.

133. Rao RN, Naidu CG, Prasad KG and Narasimha R. Development and validation of a RP-HPLC method for stability-indicating assay of gemifloxacin mesylate including identification of related substances by LC-ESI-MS/MS, $1 \mathrm{H}$ and 13C NMR spectroscopy. Biomed Chromatogr. 2011;25(11):12221229. http://dx.doi.org/10.1002/bmc.1594 ; PMid:21370250.

134. Bedse G, Kumar V and Singh S. Study of forced decomposition behavior of lamivudine using LC, LC-MS/TOF and MSn. J Pharm Biomed Anal. 2009;49(1):55-63. http://dx.doi.org/10.1016/j.jpba.2008.10.002; PMid:19022602.

135. Krishnaiah C, Murthy MV, Kumar R and Mukkanti K. Development of a stability-indicating UPLC method for determining olanzapine and its associated degradation products present in active pharmaceutical ingredients and pharmaceutical dosage forms. J Pharm Biomed Anal. 2011;54(4):667-673. http://dx.doi.org/10.1016/j.jpba.2010.10.013; PMid:21075576.

136. Sawant SD and Barge VU. Identification and characterization of forced degradation products of paliperidone using LC-APCI-Ion Trap-MS. J Pharm Res. 2013; 6:39-47. http://dx.doi.org/10.1016/j.jopr.2012.11.011.

137. Pawar SM, Khatal LD, Gabhe SY and Dhaneshwar SR. Establishment of inherent stability of pramipexole and development of validated stability indicating LC-UV and LC-MS method. J Pharm Anal 2013;3(2):109-117. http://dx.doi.org/10.1016/j.jpha.2012.07.011.

138. Namdev D, Borkar RN, Raju B, Kalariya PD, Rahangdale V, Gananadhamu S et al., Identification of forced degradation products of tamsulosin using liquid chromatography/electrospray ionization tandem mass spectrometry. J Pharm Biomed Anal. 2014;88:245-255. http://dx.doi.org/10.1016/j.jpba.2013.08.052; PMid:24083958
139. Sawant S and Barge V. A Validated Stability Indicating RP-HPLC Method for Valganciclovir, Identification and Characterization of Forced Degradation Products of Valganciclovir Using LC-MS/MS. Acta Chromatogr. 2014;26(1):29-42. http://dx.doi.org/10.1556/AChrom.26.2014.1.4

140. Dunge A, Sharda N, Singh B and Singh S. Validated specific HPLC method for determination of zidovudine during stability studies. J Pharm Biomed Anal. 2005;37(5):1109-1114. http://dx.doi.org/10.1016/j.jpba.2004.09.013; PMid:15862693.

141. Elzayat EM, Ibrahim MF, Abdel-Rahman AA, Ahmed SM, Alanazi FK, et al. A validated stability-indicating UPLC method for determination of diclofenac sodium in its pure form and matrix formulations, Arab J Chem (in-press) http://dx.doi.org/10.1016/j.arabjc. 2013.12.022.

142. Alarfaj NA and Aly FA. Micelle-enhanced spectrofluorimetric method for determination of cholesterol-reducing drug ezetimibe in dosage forms. J Fluoresc. 2012;22(1):9-15. http://dx.doi.org/10.1007/s10895-011-0952-x; PMid:21858740

143. Mahadik M, Bhusari V, Kulkarni M and Dhaneshwar S. LC-UV and LC-MS evaluation of stress degradation behavior of tenatoprazole,. J Pharm Biomed Anal. 2009;50(5):787-793. http://dx.doi.org/10.1016/j.jpba.2009.06.026; PMid:19615841

144. Pavia DL. Lampman GM, Kriz GS. Introduction to spectroscopy. 3rd ed. Philadelphia: Harcourt College Publishers; 2001.

145. Kalariya PD, Raju B, Borkar RM, Namdev D, Gananadhamu S, Nandekar PP et al. Characterization of forced degradation products of ketorolac tromethamine using LC/ESI/Q/TOF/MS/MS and in silico toxicity prediction. J Mass Spectrom. 2014;49(5):380-391. http://dx.doi.org/10.1002/jms.3351; PMid:24809899.

146. Kalgutkar AS, Nguyen HT, Vaz ADN, Doan A, Dalvie DK, McLeod DG et al. In vitro metabolism studies on the isoxazole ring scission in the antiinflammatory agent leflunomide to its active-cyanoenol metabolite a771726: mechanistic similarities with the cytochrome p450-catalyzed dehydration of aldoximes. Drug Metab Dispos. 2003;31(10):1240-1250. http://dx.doi. org/10.1124/dmd.31.10.1240; PMid:12975333

147. Saini $B$ and Bansal $G$. Isolation and characterization of a degradation product in leflunomide and a validated selective stability-indicating HPLC-UV method for their quantification. J Pharm Anal (in-press) http://dx.doi.org/10.1016/j. jpha.2014.10.003. http://dx.doi.org/10.1016/j.jpha.2014.10.003.

148. Siddiqui MR, Alothman ZA and Wabaidur SM. Ultra performance liquid chromatography-mass spectrometric method for determination of ampicillin and characterization of its forced degradation products. J Chromatogr Sci. 2014;52(1):1273-1280. http://dx.doi.org/10.1093/chromsci/bmt211; PMid:24474427

149. Tiwari R, Bonde C and Bothara K. LC-MS/TOF and MSn studies on forced degradation behavior of flucloxacillin and development of a validated stability indicating LC method. Acta Chromatogr. 2014;26(1):43-55. http://dx.doi. org/10.1556/AChrom.26.2014.1.5

\section{SUMMARY}

- Hydrolysis is the major route of degradation of drugs leading to formation of impurities and/or degradation products. The rate and extent of hydrolytic degradation is governed by the nature of functional groups present in drug molecule.

- Hydrolysis of amide, ester and ether linkages and of nitrile, hydroxyl and amino groups is very well known and mechanisms are well established in literature.

- Many drugs also degrade under hydrolytic conditions through unconventional reactions such as oxidation, base catalyzed ether hydrolysis, coupling, N-alkylation, C-C bond cleavage, dehalogenation, hydroxylation, dehydration of carboxylic acids, deamination, cyclization, dehydrogenation and decarboxylation.

- Mechanisms of most of such unconventional hydrolytic reactions are not reported or proposed in literature.

- Herein, we have critically analysed various such reports on unconventional hydrolytic routes of degradation and proposed the most probable mechanisms of these reactions with support from the standard literature.

- These data will assist an analyst to predict all possible conventional as well as unconventional hydrolytic degradation products/impurities of a drug during forced degradation studies. 